Canadian Oncology

Nursing Journal

Revue canadienne

de soins infirmiers

en oncologie

Volume 31, Issue 1 • Winter 2021

elSSN: 2368-8076 


\title{
Diminuer le recours au service des urgences pour les patients externes présentant des symptômes aigus de cancer : revue intégrative sur l'émergence des centres de soins d'urgence en cancérologie
}

\author{
par Tammy L. Patel, Shelley Raffin Bouchal, Catherine M. Laing et Stéphanie Hubbard
}

\section{RÉSUMÉ}

Cette revue intégrative de la littérature avait pour but de cerner les possibilités de recherche en sciences infirmières concernant la gestion des symptômes aigus $d u$ cancer des patients externes dans les centres de soins d'urgence en cancérologie. Les patients aux prises avec des symptômes aigus du cancer (ex. fièvre, troubles gastro-intestinaux ou douleur non soulagée) venant d'un milieu de soins externes sollicitent principalement sur les services des urgences pour se faire évaluer et traiter. Cependant, ce modèle de soins n'est plus viable et fait ressortir les inefficacités du système de soins de santé. Les centres de soins d'urgence en cancérologie permettent aux patients qui présentent ces symptômes d'être traités par des spécialistes en oncologie au sein de centres de cancérologie pour patients externes. Malheureusement, les rares travaux de recherche sur les centres de soins d'urgence en cancérologie, tant sur le plan opérationnel qu'expérimental, rendent difficile l'adoption de ce nouveau modèle de soins par d'autres. Voici les questions centrales qui ont guidé cette revue intégrative : 1) Quel est l'état de la science concernant les centres de soins d'urgence en cancérologie, et quelles sont les différences si on les compare aux services d'urgences en matière

\section{AUTEURES}

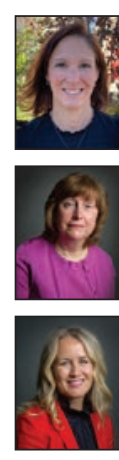

Tammy L. Patel, inf. aut., étudiante M.Sc.inf., Faculté des sciences infirmières, Université de Calgary et Tom Baker Cancer Centre, Alberta Health Services

Shelley Raffin Bouchal, inf. aut., Ph.D., professeure agrégée, Faculté des sciences infirmières, Université de Calgary

Catherine M. Laing, inf. aut. Ph.D., professeure agrégée et doyenne associée, Faculté des sciences infirmières, Université de Calgary

Stéphanie Hubbard, inf. aut., M.Sc.inf., IP, Tom Baker Cancer Centre, Alberta Health Services

Toute correspondance relative à cet article doit être adressée à : Tammy L. Patel, a/s Shelley Raffin Bouchal, professeure agrégée, Faculté des sciences infirmières, Université de Calgary, Professional Faculties Building 2270, 2500 University Dr NW, Calgary, AB T2N $1 \mathrm{~N} 4$.

Courriel : tammy.patel@ucalgary.ca

DOI:10.5737/236880763113650 de traitement prodigué aux patients externes présentant des symptômes aigus du cancer? 2) Où sont les centres de soins d'urgences en cancérologie dans le monde, et que sait-on de leurs activités cliniques et modèles de dotation?

Mots-clés : centres de soins d'urgence en cancérologie, soins externes en oncologie, cancer, symptômes

$\mathcal{C}$ omme indiqué dans la littérature, une grande partie des coûts liés aux soins de santé en cancérologie est associée aux visites non prévues à l'urgence et aux hospitalisations qui en découlent (Colligan et al., 2017; Hassett, O’Malley, Pakes, Newhouse, et Earle, 2006; McKenzie et al., 2011). Aux États-Unis, on estime que $3 \%$ du total des visites à l'urgence annuellement (soit 1 à 3 millions de visites) sont liées au cancer (Cooksley et Rice, 2017); et selon un examen systématique de 18 études effectuées par Vandyk, Harrison, Macartney, RossWhite et Stacey (2012), plus de la moitié de ces consultations se termineront par une hospitalisation. Une étude similaire à grande échelle sur le cancer du sein aux États-Unis a révélé que $51 \%$ des patientes seront hospitalisées une fois ou consulteront une fois à l'urgence pendant la première année de traitement (Hassett et al., 2006). Une étude menée à Calgary a fait état de taux d'utilisation similaires de l'urgence par les patients atteints de cancer; 1881 patients ont consulté 4623 fois à l'urgence de juillet 2007 à septembre 2011 (Prystajecky et al., 2012). De plus, la charge croissante des dépenses en soins de santé dans le monde s'explique notamment par l'amélioration des taux de survie au cancer (Young et al., 2016).

Compte tenu de l'augmentation annuelle des diagnostics de cancer et des dépenses liées aux consultations imprévues à l'urgence et aux hospitalisations, l'innovation dans la prestation de soins rentables aux patients est impérative. Au Canada, le taux de diagnostics de cancer au cours des 35 dernières années est passé de 89200 en 1984 à 220200 en 2019, une augmentation annuelle moyenne de 7 \% (Comité consultatif canadien sur les statistiques du cancer, 2019). Les États-Unis ont fait état de plus de 499000 visites à l'urgence liées au cancer en 2007, pour un total de 777,24 M\$ US, soit un coût moyen de 1558 \$ par visite à l'urgence (Mayer, Travers, Wyss, Leak et Waller, 2011). Également aux États-Unis, « une hospitalisation de 2 à 3 jours avec perfusion et antiémétiques coûte environ 8729 \$ US » (Mason, DeRubeis, Foster, Taylor et Worden, 2013, p. 585). En revanche, un patient externe qui consulte une infirmière praticienne, avec perfusion et prise d'antiémétiques ne coûte en moyenne que 197 US\$ (Mason et al., 2013). 
Les ressources des services d'urgence (c'est-à-dire l'établissement, le personnel et le temps) restent fixes, ce qui peut amener les patients immunodéprimés atteints d'un cancer à faire face à des temps d'attente prolongés et à un risque accru d'exposition à des agents pathogènes. De même, l'échelle de triage et de gravité (ÉTG) conventionnelle des services d'urgence canadiens ne saisit pas avec précision les besoins particuliers de ces patients, comme le montre le tableau 1. Par exemple, la fièvre doit être traitée comme une urgence médicale chez les patients atteints de cancer, mais l'ÉTG assigne un niveau trois à la fièvre (sur une échelle d'urgence de un à cinq) avec des temps d'attente idéaux allant jusqu'à 30 minutes; ce qui peut exposer ces patients à un risque accru de septicémie et de décès (Cooksley et Rice, 2017; Trip, Boloorchi et Berman, 2011). De nombreuses études ont établi les symptômes les plus fréquents des patients atteints de cancer qui se présentent à l'urgence : douleur, fièvre, symptômes respiratoires, troubles gastro-intestinaux (Clarke, Bird, Kakuchi, Littlewood et van Hamel Parsons, 2015; Cooksley et Rice, 2017; Hassett et al., 2006; Livingston, Craike et Considine, 2011;

\begin{tabular}{|l|l|l|}
\hline $\begin{array}{l}\text { Tableau 1. Échelle canadienne de triage et de gravité pour les } \\
\text { départements des urgences (ÉTG) }\end{array}$ \\
\hline $\begin{array}{l}\text { Échelle canadienne } \\
\text { de triage et de } \\
\text { gravité (ÉTG) }\end{array}$ & $\begin{array}{l}\text { Délai de prise } \\
\text { en charge } \\
\text { thérapeutique }\end{array}$ & Définitions et exemples \\
\hline 1 - Réanimation & Immédiatement & $\begin{array}{l}\text { État qui constitue une menace } \\
\text { pour la vie ou pour un membre } \\
\text { (ex. arrêt cardiaque, trauma } \\
\text { majeur) }\end{array}$ \\
\hline 2 - Très urgent & $<15$ minutes & $\begin{array}{l}\text { État qui constitue une menace } \\
\text { potentielle pour la vie ou pour } \\
\text { un membre (ex. compression } \\
\text { de la moelle épinière, douleur } \\
\text { thoracique) }\end{array}$ \\
\hline 3 - Urgent & $<30$ minutes & $\begin{array}{l}\text { État qui pourrait évoluer vers } \\
\text { un problème grave nécessitant } \\
\text { une intervention d'urgence } \\
\text { (ex. détresse respiratoire légère, } \\
\text { vomissements et/ou nausées) }\end{array}$ \\
\hline $4-$ Moins urgent & $<60$ minutes & $\begin{array}{l}\text { État qui bénéficierait d'une } \\
\text { intervention médicale ou d'un } \\
\text { réconfort dans un délai d'une à } \\
\text { deux heures (ex. infection des } \\
\text { voies urinaires, constipation) }\end{array}$ \\
\hline health network experience ». Par K. Trip, A. Boloorchi, \& H. Berman, \\
\hline - Non urgent
\end{tabular}

Majem et al., 2007; Mayer et al., 2011; McKenzie et al., 2011; Meisenberg, Graze et Brady-Copertino, 2014; Prystajecky et al., 2012; Ruegg, 2013; Sitzes-Determann, 2009; van der Meer et al., 2016; Vandyk et al., 2012). La nature constante, prévisible et non urgente de ces symptômes indique qu'on pourrait améliorer les services aux patients externes et ainsi réduire les visites à l'urgence.

\section{TRAITEMENT DES PATIENTS EXTERNES PRÉSENTANT DES SYMPTÔMES AIGUS DU CANCER}

Dans les centres de soins externes en cancérologie, trois stratégies servent principalement à gérer les symptômes liés au cancer : l'éducation du patient, la prévention et le soutien téléphonique (Young et al., 2016). Malheureusement, les symptômes aigus du cancer (fièvre, troubles gastro-intestinaux, douleur non soulagée, etc.) sont parfois trop graves pour que les soins autoadministrés à la maison fonctionnent et requièrent des soins médicaux d'urgence. Par le passé, sur tous les continents, les centres de soins externes en cancérologie comptent sur les services d'urgence pour répondre aux besoins urgents et non comblés des patients atteints de cancer (Young et al., 2016). Comme l'ont démontré Prystajecky et al. (2012), la mise en place d'un soutien téléphonique jour et nuit au centre de soins externes en cancérologie de Calgary a été associée à une diminution importante (de $32 \%$ ) des visites à l'urgence sur une période quatre mois. Mais malgré ce soutien téléphonique menant à une baisse du nombre de visites à l'urgence, un nombre considérable de patients cancéreux ont besoin de soins médicaux en personne (Prystajecky et al., 2012).

Tel qu'indiqué par Colligan et collègues (2017), « 19 à $23 \%$ des visites à l'urgence et des hospitalisations de patients cancéreux sont potentiellement évitables » (p. 873) et les symptômes présentés par ces patients seraient mieux traités dans un contexte ambulatoire. Les plus récents centres de soins d'urgence en cancérologie sont spécialement conçus pour les patients externes présentant des symptômes aigus du cancer non urgents, et offrent ce milieu de soins externes alternatif. Ces centres sont commodément situés dans les centres de soins externes en cancérologie actuels, avec le double objectif de désengorger les urgences et de faire en sorte que les besoins en cancérologie soient comblés en un seul endroit par des spécialistes en oncologie. De plus, les centres de soins urgents en cancérologie permettent aux patients cancéreux d'être mieux triés, en fonction de l'acuité de l'urgence oncologique, ce qui améliore la sécurité des patients et permet une approche des soins centrée sur le patient. Les avantages des centres de soins d'urgence en cancérologie encouragent l'adoption de ce nouveau modèle de soins complets tout en mettant fin à la dépendance aux services des urgences des centres de soins externes en cancérologie, dépendance qui devrait être dépassée.

\section{BUT DE L'ÉTUDE}

L'objectif de cette revue intégrative de la littérature était de déterminer l'étendue et les types de recherches menées pour comparer les centres de soins d'urgences en cancérologie et les 
services des urgences dans le traitement des patients externes présentant des symptômes aigus du cancer. Une bonne connaissance de la littérature permet de repérer les connaissances manquantes et les sujets de recherche potentiels. Plus précisément, cet examen a servi de base à la formulation d'une question de recherche en soins infirmiers de deuxième cycle sur les centres de soins d'urgence en oncologie.

\section{MÉTHODOLOGIE}

Une revue intégrative de la littérature a été lancée pour aider à déterminer la pertinence selon l'expérience passée de délaisser le modèle de soins des services d'urgence pour les patients en oncologie au profit d'un modèle global de soins avec des centres de soins d'urgence en cancérologie. La revue intégrative offre une approche alternative aux chercheurs qui étudient de vastes sujets ou examinent des questions de recherche tout en permettant l'inclusion simultanée de divers modèles d'étude et types d'articles - une considération importante pour la pratique des soins infirmiers basée sur les données probantes (Whittemore et Knafl, 2005). La synthèse des études expérimentales et non expérimentales contribue à la pleine compréhension du phénomène étudié (Whittemore et Knafl, 2005). En outre, « la multiplicité des objectifs peut donner lieu à une représentation complète de concepts, de théories ou de problèmes de santé complexes et importants en soins infirmiers » (Whittemore et Knafl, 2005, p. 548). La méthodologie décrite dans le cadre d'une revue intégrative de Whittemore et Knafl (2005) comprend cinq étapes : 1) formulation du problème; 2) recherche documentaire; 3) évaluation des données; 4) analyse des données; 5) présentation des données.

\section{Formulation du problème}

Voici les questions centrales de recherche qui ont guidé cette revue intégrative de la littérature :

1. Quel est l'état de la science concernant les centres de soins d'urgence en cancérologie, et quelles sont les différences si on les compare aux services d'urgences en matière de traitement prodigué aux patients externes présentant des symptômes aigus du cancer?

2. Où sont les centres de soins d'urgences en cancérologie dans le monde, et que sait-on de leurs activités cliniques et modèles de dotation?

\section{Recherche documentaire}

En consultation avec un bibliothécaire de recherche et un rédacteur scientifique - pour l'accompagnement en stratégie documentaire, interface et navigation dans les bases de données, et utilisation d'Endnote - la première auteure a élaboré et utilisé une stratégie de recherche structurée, avec schématisation des termes de recherche dans les titres, utilisation de troncatures et de caractères jokers, et recours aux opérateurs booléens dans chaque base de données. Après plusieurs recherches itératives initiales, les termes de recherche suivants ont été utilisés avec succès pour chaque interface de données : (« oncologie » OU « cancer ») ET (« urgence » OU « aigu » $\mathrm{OU}$ « urgent » $\mathrm{OU}$ « rapide ») ET (« clinique » $\mathrm{OU}$ « centre » $\mathrm{OU}$ « service ») ET (« traitement » $\mathrm{OU}$ « soins » $\mathrm{OU}$ « prise en charge » ET (« patient externe » OU « ambulatoire » OU « complet » OU « communauté ») ET « symptôme* ».1

Toute la littérature a été recensée à l'aide d'une variété de sources, y compris des bases de données électroniques et des listes de références, ainsi que de la littérature grise provenant de ProQuest, Google Scholar et d'Internet. La stratégie de recherche a évité les restrictions de date puisque le sujet d'étude n'est apparu que récemment. L'examen intégratif a consisté en des recherches dans quatre bases de données en ligne : CINAHL $(n=127)$, MEDLINE $(n=704)$, PubMed ( $n=907)$ et ProQuest Theses \& Dissertations Global $(n=13)$. La recherche la plus récente a été effectuée en octobre 2018.

La documentation obtenue grâce à cette stratégie de recherche a été examinée par la première auteure comme l'exige son programme d'études supérieures en sciences infirmières. Comme le résume la figure 1 , la stratégie de recherche dans la base de données en ligne a permis de recenser 1738 articles tandis que d'autres sources ont révélé d'autres articles pertinents $(n=16)$, y compris les ressources en ligne et les listes de références parcourues, menant donc à un total de 1754 sources documentaires. Les doublons $(n=35)$ ont été éliminés lors de la vérification des autres titres et des résumés des articles, puis les titres et résumés restants $(n=1719)$ ont fait l'objet d'une analyse d'adéquation sur la base des critères d'inclusion et d'exclusion décrits ci-dessous. Il en est resté 66 articles en texte intégral, qui ont été évalués pour en déterminer l'admissibilité. Au total, 17 sources documentaires ont finalement été incluses dans la revue intégrative comme répondant adéquatement aux questions essentielles et respectant tous les critères d'inclusion.

Pour déterminer les sources pertinentes, voici quels ont été les critères d'inclusion d'un article dans cette revue intégrative : 1) publications évaluées par des pairs dont le texte intégral est disponible; 2) études publiées en anglais; 3) études axées sur l'utilisation soit des services des urgences ou des centres de soins d'urgence en cancérologie pour le traitement des patients présentant des symptômes aigus du cancer. Des critères d'exclusion finaux sont cependant apparus lors de l'examen systématique des articles en texte intégral. Des articles ont donc été exclus sur la base des critères suivants : 1) études axées sur les patients en soins palliatifs atteints d'un cancer avancé; 2) études portant sur les patients en pédiatrie atteints de cancer; 3) études axées sur les processus des services des urgences ou des services aux collectivités (soins à domicile) pour les patients cancéreux, étant donné que ces processus ne sont pas généralisables au système de santé canadien. .

\section{Évaluation des données}

Chaque source documentaire répondant aux critères d'inclusion et abordant une question centrale de recherche a fait l'objet d'une évaluation critique par la première auteure à l'aide des deux listes de vérification de Specialist Unit for Review of

1 Recherche effectuée en anglais : ("oncology" OR “cancer”) AND ("emergency" OR "acute" OR "urgent" OR "rapid") AND ("clinic" OR “center” OR “centre” OR “department”) AND (“treatment” OR "care" OR "management") AND ("outpatient" OR "ambulatory" OR "comprehensive” OR “community”) AND “symptom*” 
Figure I. Diagramme fonctionnel de la stratégie de recherche

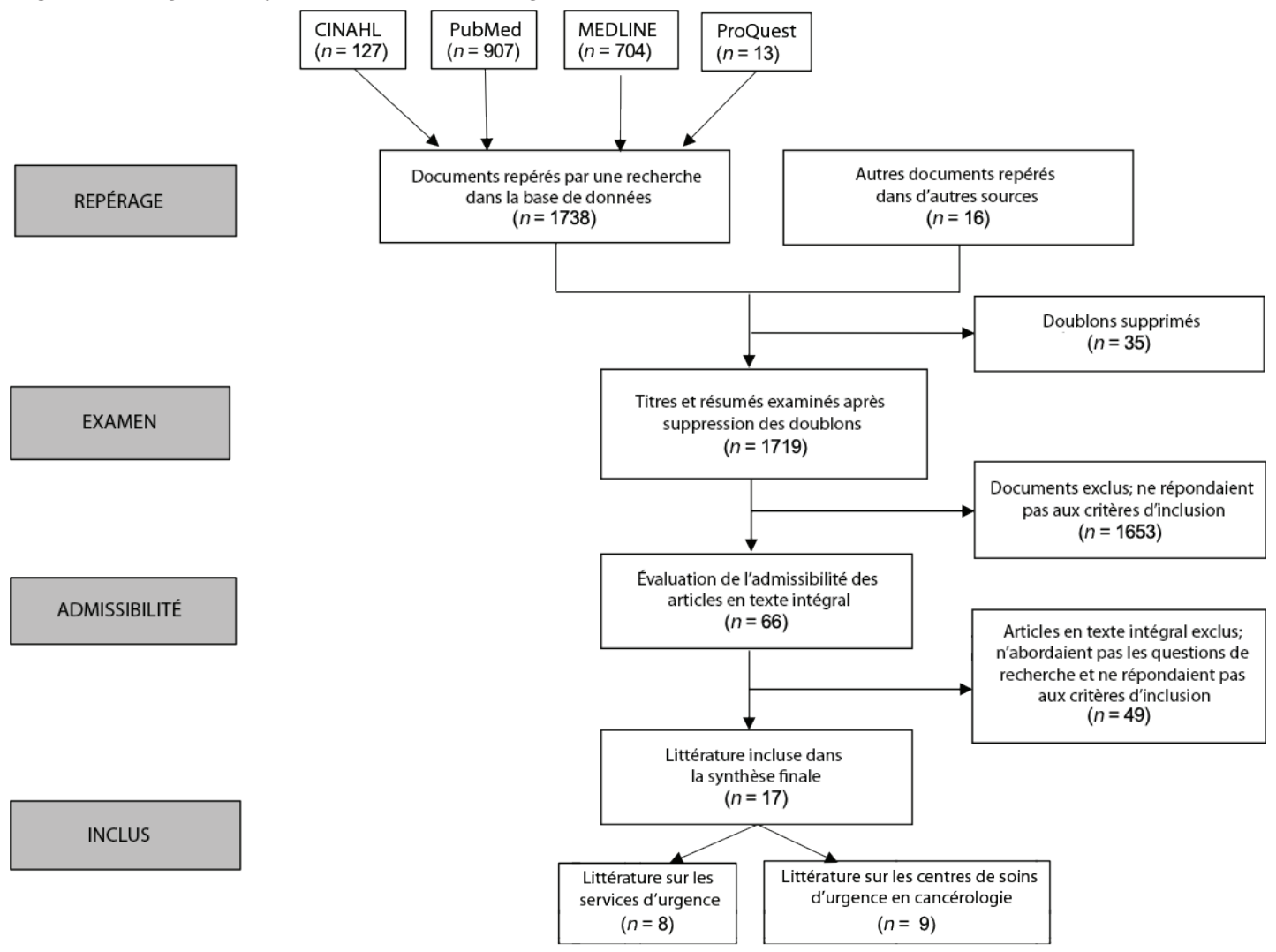

Evidence (SURE) (2018a, 2018b) contenant les critères présentés dans les tableaux 2 et 3 . Les 17 sources documentaires finales ont été divisées en deux tableaux de vérification spécifiques aux études de cohorte qualitatives (tableau 2) ou quantitatives (tableau 3). L'endroit des soins de santé (c.-à-d. service d'urgence versus centre de soins d'urgence en oncologie) de chaque étude a également été mis en évidence dans chaque tableau. Chaque source documentaire a été évaluée en fonction de la clarté du but de l'étude, de sa qualité méthodologique, de sa précision et de sa validité externe. Bien que chaque étude ait eu des limites, la plupart d'entre elles ont été classées de qualité moyenne à élevée et aucune n'a été exclue. Les études de moindre qualité ont été citées moins fréquemment pour tenir compte de leur moindre validité externe. Il a été reconnu par Whittemore et Knafl (2005) que « l'évaluation de la qualité des sources primaires dans le cadre d'une revue intégrative est complexe » (p. 550). Par conséquent, les outils d'évaluation critique choisis pour cet examen visaient à garantir l'applicabilité de chaque liste de vérification à tous les modèles deétude tout en restant pertinents pour la littérature grise incluse.

\section{Analyse des données}

L'objectif de cette revue était de mieux comprendre les centres de soins d'urgence en cancérologie en tant que milieu de soins externes potentiel pouvant présenter une alternative aux services des urgences pour le traitement des patients externes aux prises avec des symptômes aigus du cancer. Chaque article en texte intégral a été lu pour extraire et enregistrer des données détaillées dans une feuille de calcul Excel standardisée. Pour améliorer la clarté des informations et faciliter l'analyse,

\begin{tabular}{|l|l|l|}
\hline \multicolumn{3}{|l|}{ Tableau 2. Évaluation critique des études qualitatives } \\
\hline & $\begin{array}{l}\text { Étude } \\
\text { qualitative }\end{array}$ & $\begin{array}{l}\text { Littérature } \\
\text { grise }\end{array}$ \\
\hline Étude & $\begin{array}{l}\text { Clarke } \\
\text { et al. } \\
\text { (2015) }\end{array}$ & $\begin{array}{l}\text { Sitzes- } \\
\text { Determann } \\
\text { (2009) }\end{array}$ \\
\hline Liste de vérification & & \\
\hline $\begin{array}{l}\text { L'étude porte-t-elle sur une question/hypothèse } \\
\text { clairement ciblée? }\end{array}$ & Oui & Oui \\
\hline Le choix de la méthode qualitative est-il approprié? & Oui & Oui \\
\hline $\begin{array}{l}\text { La stratégie d'échantillonnage est-elle } \\
\text { clairement décrite? }\end{array}$ & Oui & Oui \\
\hline $\begin{array}{l}\text { La relation entre le ou les chercheurs et les } \\
\text { participants est-elle explorée? }\end{array}$ & Non & Non \\
\hline Les questions éthiques sont-elles abordées? & Oui & Oui \\
\hline $\begin{array}{l}\text { Le processus d'analyse/interprétation des } \\
\text { données est-il décrit et justifié? }\end{array}$ & Oui & Oui \\
\hline Les résultats sont-ils crédibles? & Oui & Oui \\
\hline $\begin{array}{l}\text { Des parrainages/conflits d'intérêts sont-ils } \\
\text { signalés? }\end{array}$ & Non & Non \\
\hline Les auteurs ont-ils identifié des limites? & Oui & Oui \\
\hline $\begin{array}{l}\text { Littérature sur les services des urgences } \\
\text { Littérature sur les centres de soins d'urgence en oncologie }\end{array}$ \\
\hline $\begin{array}{l}\text { Note : Adapté de « Questions to assist with the critical appraisal of } \\
\text { Unalitative studies ", de Specialist Unit for Review Evidence (SURE), 2018, } \\
\text { SURE-CA-form-for-Qualitative_2018.pdf }\end{array}$ & \\
\hline
\end{tabular}




\begin{tabular}{|c|c|c|c|c|c|c|c|c|c|c|c|c|c|c|c|}
\hline \multirow[b]{2}{*}{ Liste de vérification } & \multicolumn{13}{|c|}{ Étude qualitative } & \multicolumn{2}{|c|}{\begin{tabular}{|l} 
Littérature \\
grise
\end{tabular}} \\
\hline & 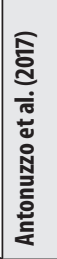 & 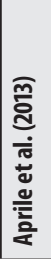 & 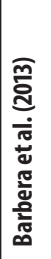 & 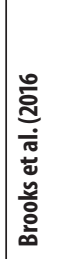 & 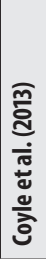 & 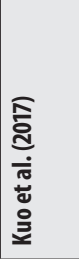 & 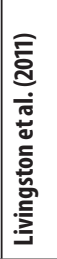 & 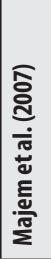 & 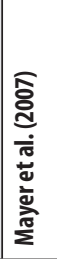 & 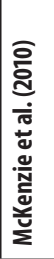 & 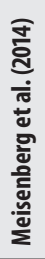 & 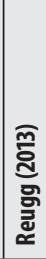 & 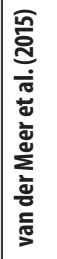 & 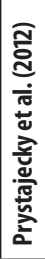 & 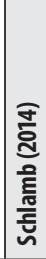 \\
\hline Le devis de l'étude est-il clairement énoncé? & Oui & Oui & Oui & Oui & Non & Oui & Oui & Non & Non & Oui & Oui & Non & Oui & Oui & Non \\
\hline L'étude répond-elle sur une question clairement cernée? & Oui & Oui & Oui & Oui & Oui & Non & Oui & Non & Oui & Oui & Oui & Non & Oui & Oui & Non \\
\hline Le milieu, les lieux et les dates utiles sont-ils fournis? & Oui & Oui & Oui & Oui & Oui & Oui & Oui & Oui & Oui & Oui & Oui & Oui & Oui & Oui & Oui \\
\hline Les participants ont-ils été sélectionnés de manière équitable? & Oui & Oui & Oui & Oui & Oui & Oui & Oui & Oui & Oui & Oui & Oui & Oui & Oui & $\begin{array}{l}\mathrm{Ne} \\
\text { sait } \\
\text { pas }\end{array}$ & $\begin{array}{l}\mathrm{Ne} \\
\text { sait } \\
\text { pas }\end{array}$ \\
\hline Les caractéristiques des participants sont-elles fournies? & Oui & Oui & Oui & Oui & Non & Oui & Oui & Non & Oui & Oui & Oui & Non & Oui & Non & Non \\
\hline Les mesures de l'exposition et des résultats sont-elles appropriées? & Oui & Oui & Oui & Oui & Oui & Oui & Oui & Oui & Oui & Oui & Oui & Oui & Oui & Oui & Oui \\
\hline Le biais a-t-il été pris en compte? & $\begin{array}{l}\mathrm{Ne} \\
\text { sait } \\
\text { pas }\end{array}$ & Oui & Oui & \begin{tabular}{|l}
$\mathrm{Ne}$ \\
sait \\
pas
\end{tabular} & $\begin{array}{l}\mathrm{Ne} \\
\text { sait } \\
\text { pas }\end{array}$ & $\begin{array}{l}\text { Oui Ne } \\
\text { sait pas }\end{array}$ & Oui & \begin{tabular}{|l}
$\mathrm{Ne}$ \\
sait \\
pas
\end{tabular} & Oui & Oui & $\begin{array}{l}\mathrm{Ne} \\
\text { sait } \\
\text { pas }\end{array}$ & Non & Oui & $\begin{array}{l}\mathrm{Ne} \\
\text { sait } \\
\text { pas }\end{array}$ & Non \\
\hline Ya-t-il une description de la manière dont on est parvenu à la taille de l'étude? & Non & Non & Non & Non & Non & Non & Oui & Non & Non & Non & Non & Non & Non & Non & Non \\
\hline Les méthodes statistiques sont-elles bien décrites? & Non & Oui & Oui & Oui & Non & Oui & Oui & Non & Oui & Oui & Non & Non & Oui & Non & Non \\
\hline Des informations sont-elles fournies sur le flux des participants? & Non & Oui & Oui & Oui & Non & Non & Oui & Oui & Non & Oui & Non & Non & Non & Non & Non \\
\hline Les résultats sont-ils bien décrits? & Oui & Oui & Oui & Oui & Non & Oui & Oui & Oui & Oui & Oui & Oui & Oui & Oui & Oui & Non \\
\hline Des parrainages/conflits d'intérêts sont-ils signalés? & Non & Non & Non & Non & Non & Non & Non & Non & Non & Non & Non & Non & Non & $\begin{array}{l}\mathrm{Ne} \\
\text { sait } \\
\text { pas }\end{array}$ & $\begin{array}{l}\mathrm{Ne} \\
\text { sait } \\
\text { pas }\end{array}$ \\
\hline Les auteurs ont-ils bien identifié des limites et, si oui, sont-elles reprises ci-dessus? & Non & Oui & Oui & Oui & Non & Oui & Non & Oui & Oui & Non & Oui & Oui & Oui & Non & Non \\
\hline
\end{tabular}

\begin{tabular}{|l|}
\hline Littérature sur les services des urgences \\
\hline Littérature sur les centres de soins d'urgence en oncologie \\
\hline
\end{tabular}

Note : Adapté de « Questions to assist with the critical appraisal of qualitative studies », de Specialist Unit for Review Evidence (SURE), 2018,

URL : https://www.cardiff.ac.uk/__data/assets/pdf_file/0007/1142971/SURE-CA-form-for-Qualitative_2018.pdf

les données ont été divisées en deux sous-groupes (Whittemore et Knafl, 2005). Ces sous-groupes sont chacun représentés par des tableaux d'extraction de données séparés, l'un incluant toutes les sources documentaires sur les services des urgences (tableau 4), et l'autre incluant toutes les sources documentaires sur les centres de soins d'urgence en cancérologie (tableau 5). Les données d'étude extraites de la littérature sur les services des urgences ont été résumées en fonction des auteurs (année) et du lieu, du but de l'étude, de son devis, de l'échantillon, des résultats et constats, des activités et de la dotation en personnel, ainsi que des limites. Les thèmes récurrents ont ensuite été déterminés à partir des données extraites, à l'aide de méthodes de comparaison itératives, puis reformulés pour obtenir la synthèse finale des résultats (Whittemore et Knafl, 2005). Ce processus a été effectué par la première auteure et revu par la directrice de thèse (la deuxième auteure). Les données des deux tableaux d'extraction ont été comparées et contrastées pour établir les modèles et les thèmes les plus fréquents afin de répondre à la première question centrale. La synthèse thématique finale des résultats est présentée ci-dessous sous la forme de trois thèmes principaux et de sept sous-thèmes.

\section{PRÉSENTATION DES DONNÉES}

\section{Résultats de recherche}

Les 17 sources documentaires incluses dans cette revue intégrative faisaient preuve d’une grande hétérogénéité en ce qui concerne la méthodologie de l'étude et les axes de recherche (Clarke et al., 2015; Sitzes-Determann, 2009). La majorité $(n=12)$ s'appuyait sur des méthodes de collecte de données rétrospectives (Antonuzzo et al., 2017; Aprile et al., 2013; Barbera et al., 2013; Kuo, De Silva, Diwakarla et Yip, 2017; Livingston et al., 2011; Majem et al., 2007; Mayer et al., 2011; McKenzie et al., 2011; Meisenberg et al., 2014; Ruegg, 2013; Schlamb, 2014; van der Meer et al., 2016). Huit études portaient sur les modes d'utilisation des services des urgences (Barbera et al., 2013; Brooks et al., 2016; Clarke et al., 2015; Livingston et al., 2011; Mayer et al., 2011; McKenzie et al., 2011; Prystajecky et al., 2012; van der Meer et al., 2016); les neuf autres se concentraient spécifiquement sur les centres de soins d'urgence en cancérologie (Antonuzzo et al., 2017; Aprile et al., 2013; Coyle, Miller et Paulson, 2013; Kuo et al., 2017; Majem et al., 2007; Meisenberg et al., 2014; Ruegg, 2013; Schlamb, 2014; Sitzes-Determann, 2009). Six études ont été réalisées aux États-Unis (Brooks et al., 2016; Coyle et al., 2013; Mayer et al., 2011; Meisenberg et al., 2014; 


\begin{tabular}{|c|c|c|c|c|c|}
\hline \multicolumn{6}{|c|}{ Littérature sur les services des urgences } \\
\hline $\begin{array}{l}\text { Auteurs } \\
\text { (année) } \\
\text { Endroit }\end{array}$ & But de l'étude & Devis de l'étude & Échantillon & Résultats de l'étude & Limites de l'étude \\
\hline $\begin{array}{l}\text { Barbera } \\
\text { et al. } \\
\text { (2013) } \\
\text { Canada }\end{array}$ & $\begin{array}{l}\text { Déterminer la relation } \\
\text { entre la gravité du score } \\
\text { de l'Échelle d'évaluation } \\
\text { des symptômes } \\
\text { d'Edmonton (ESAS) } \\
\text { et la probabilité de } \\
\text { présenter une urgence } \\
\text { dans les } 7 \text { jours suivant } \\
\text { l'évaluation. }\end{array}$ & $\begin{array}{l}\text { Étude de cohorte } \\
\text { rétrospective quantitative } \\
\text { de patients cancéreux } \\
\text { cliniques externes et } \\
\text { communautaires en } \\
\text { Ontario entre } 2007 \text { et } \\
2009 .\end{array}$ & $\begin{array}{l}n=45118 \text { patients } \\
\text { uniques. } \\
\text { Comprenait tous les } \\
\text { patients évalués entre } \\
\text { la date du diagnostic } \\
\text { et la date du décès ou } \\
\text { du dernier suivi lors de } \\
\text { leur première évaluation } \\
\text { avec un outil ESAS. } \\
\text { Comprend toutes les } \\
\text { régions de la province } \\
\text { et tout diagnostic de } \\
\text { cancer, quel que soit } \\
\text { l'âge, recevant un } \\
\text { traitement dans un but } \\
\text { curatif ou palliatif. } \\
2 \text { 693 patients exclus } \\
\text { en raison de données } \\
\text { incomplètes sur les } \\
\text { symptômes, d'un } \\
\text { numéro d'assurance } \\
\text { maladie invalide ou } \\
\text { manquant, ou de } \\
\text { l'absence de lien avec } \\
\text { le registre du cancer de } \\
\text { l'Ontario. }\end{array}$ & $\begin{array}{l}1732 \text { (3,8 \%) patients ont effectué une } \\
\text { visite à l'urgence dans les } 7 \text { jours suivant } \\
\text { l'évaluation avec l'ESAS; } 72 \% \text { des visites } \\
\text { à l'urgence ont été effectuées en semaine; } \\
52 \% \text { pendant la journée }(8-16 \mathrm{~h} \text { ). Les } \\
\text { scores ÉTG de } 1-5 \text { étaient respectivement } \\
\text { de } 1,7 \%, 28,4 \%, 51,6 \%, 14,7 \% \text { et } \\
3,6 \% .40 \% \text { ont été hospitalisés. } 1 \% \\
\text { d'augmentation des visites à l'urgence pour } \\
\text { chaque augmentation de } 5 \text { points au score } \\
\text { total de l'ESAS. Symptômes généraux } \\
\text { associés à une forte probabilité de visite à } \\
\text { l'urgence. }\end{array}$ & $\begin{array}{l}\text { Surreprésentation des } \\
\text { patients atteints de } \\
\text { cancer du poumon (dont } \\
\text { la survie est plus courte } \\
\text { que celle de l'ensemble } \\
\text { de la population atteinte } \\
\text { de cancer), ce qui reflète } \\
\text { l'effort de recrutement } \\
\text { initial pour cette initiative. } \\
\text { Impossible de déterminer } \\
\text { où un patient se trouvait } \\
\text { dans la trajectoire de sa } \\
\text { maladie. Les résultats } \\
\text { peuvent refléter les } \\
\text { effets de l'outil ESAS lui- } \\
\text { même. Aucune donnée } \\
\text { de comparaison pour les } \\
\text { patients NON évalués } \\
\text { avec l'ESAS (c.-à-d. } \\
\text { avant l'intervention, quel } \\
\text { était le taux de visites à } \\
\text { l'urgence dans les } 7 \text { jours } \\
\text { suivant l'évaluation de } \\
\text { l'absence d'ESAS?) }\end{array}$ \\
\hline $\begin{array}{l}\text { Brooks } \\
\text { et al. } \\
\text { (2016) } \\
\text { États-Unis }\end{array}$ & $\begin{array}{l}\text { Évaluer l'efficacité } \\
\text { de la réduction de } \\
\text { l'hospitalisation des } \\
\text { patients externes } \\
\text { atteints de cancer } \\
\text { nécessitant des soins } \\
\text { aigus en intégrant un } \\
\text { oncologue à l'urgence } \\
\text { pendant les heures du } \\
\text { soir (de } 17 \text { à } 23 \mathrm{~h} \text {, du } \\
\text { dimanche au vendredi) }\end{array}$ & $\begin{array}{l}\text { Conception d'études } \\
\text { quantitatives quasi } \\
\text { expérimentales } \\
\text { pré-intervention/ } \\
\text { post-intervention. } \\
\text { L'intervention pilote a } \\
\text { examiné les données } \\
5 \text { semaines avant et après } \\
\text { l'intervention. }\end{array}$ & $\begin{array}{l}n=808 \text { visites } \\
\text { admissibles à l'urgence } \\
\text { pour les tumeurs solides } \\
\text { malignes (moyenne }= \\
11,5 \text { visites à l'urgence/ } \\
\text { jour). } \\
n=390 \text { visites avant } \\
\text { l'intervention et } \\
n=418 \text { visites pendant } \\
\text { l'intervention. }\end{array}$ & $\begin{array}{l}\text { Intervention associée à une différence } \\
\text { non significative dans les hospitalisations } \\
(p=.62) \text {. } \\
\text { Une étude indique que l'implication } \\
\text { précoce des oncologues a conduit à une } \\
\text { meilleure coordination des soins pour les } \\
\text { patients cancéreux après la visite initiale à } \\
\text { l'urgence. } \\
\text { L'étude indique que } 37 \% \text { des visites à } \\
\text { l'urgence liées à l'oncologie ont eu lieu } \\
\text { entre } 8 \text { et } 16 \mathrm{~h} \text { alors que } 31 \% \text { surviennent } \\
\text { entre } 16 \text { et } 23 \mathrm{~h} \text {. }\end{array}$ & $\begin{array}{l}\text { L'intervention pilote s'est } \\
\text { déroulée sur une courte } \\
\text { période de temps. } \\
\text { Inclusion de données } \\
\text { d'évaluation subjectives } \\
\text { de nombreux oncologues. } \\
\text { Faible généralisabilité. }\end{array}$ \\
\hline $\begin{array}{l}\text { Livingston } \\
\text { et al. (2011) } \\
\text { Australie }\end{array}$ & $\begin{array}{l}\text { Comprendre } \\
\text { les principales } \\
\text { caractéristiques des } \\
\text { visites à l'urgence } \\
\text { pour les patients } \\
\text { cancéreux nouvellement } \\
\text { diagnostiqués } \\
\text { qui suivent une } \\
\text { chimiothérapie en } \\
\text { milieu de soins externes. }\end{array}$ & $\begin{array}{l}\text { Vérification rétrospective } \\
\text { quantitative des dossiers } \\
\text { de } 443 \text { patients } \\
\text { cancéreux nouvellement } \\
\text { diagnostiqués, } \\
\text { sélectionnés au hasard, } \\
\text { qui ont reçu une } \\
\text { chimiothérapie dans le } \\
\text { cadre de l'oncologie de } \\
\text { jour et qui ont consulté à } \\
\text { l'urgence en } 2007 .\end{array}$ & $\begin{array}{l}n=443 \text { dossiers de } \\
\text { patients aléatoires (total } \\
\text { de dossiers de patients } \\
\text { atteints de cancer en } \\
\text { oncologie par jour en } \\
2007=2380 ; 32,5 \% \\
{[772] \text { ont consulté à }} \\
\text { l'urgence au moins une } \\
\text { fois). Des } 443 \text {, un peu } \\
\text { plus d'hommes que de } \\
\text { femmes, et âge moyen } \\
\text { de } 61 \text { ans. }\end{array}$ & $\begin{array}{l}\text { Catégories de triage de } 443 \text { visites à } \\
\text { l'urgence : ÉTG } 1=0,5 \%, \text { ÉTG } 2=21,6 \% \text {, } \\
\text { ÉTG } 3=48,0 \% \text {, ÉTG } 4=27,5 \% \text { et } \\
\text { ÉTG } 5=2,3 \% \text {; plus de } 60 \% \text { de l'ÉTG ont } \\
\text { reçu leur congé. Le temps d'attente variait } \\
\text { de } 0,0 \text { min à } 6,8 \text { h; la durée médiane du } \\
\text { séjour à l'urgence était } 6,3 \mathrm{~h} \text {. Principale } \\
\text { plaintes : neutropénie fébrile, nausée et } \\
\text { vomissements, et douleurs abdominales. } \\
67,3 \% \text { des visites à l'urgence ont eu } \\
\text { lieu entre } 6 \mathrm{~h} \text { et } 18 \mathrm{~h} .58 \% \text { des visites à } \\
\text { l'urgence ont entraîné une hospitalisation } \\
\text { d'une durée médiane de } 4,7 \text { jours. }\end{array}$ & $\begin{array}{l}\text { Étude limitée par des } \\
\text { données réelles recueillies } \\
\text { par voie électronique. }\end{array}$ \\
\hline
\end{tabular}

continued... 


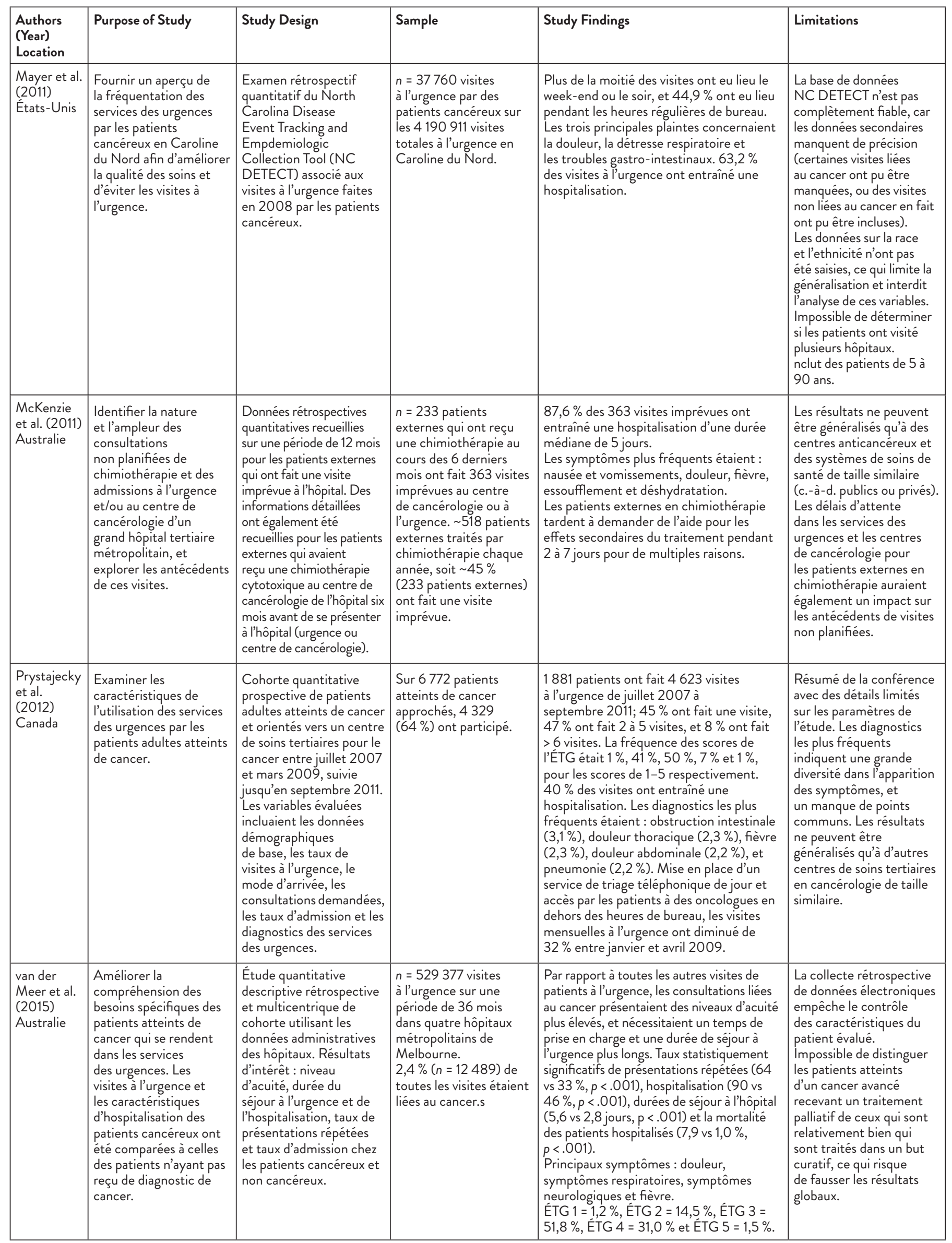




\begin{tabular}{|c|c|c|c|c|c|c|}
\hline \multicolumn{7}{|c|}{ Littérature sur les centres de soins d'urgence en oncologie } \\
\hline $\begin{array}{l}\text { Auteurs } \\
\text { (année) } \\
\text { Endroit }\end{array}$ & But de l'étude & Devis de l'étude & Échantillon & Résultats de l'étude & Activités et dotation & Limites de l'étude \\
\hline $\begin{array}{l}\text { Antonuzzo } \\
\text { et al. (2017) } \\
\text { Italie }\end{array}$ & $\begin{array}{l}\text { Déterminer si les } \\
\text { services de soins de } \\
\text { soutien ont eu un } \\
\text { impact positif sur } \\
\text { la fréquence des } \\
\text { hospitalisations non } \\
\text { planifiées. }\end{array}$ & $\begin{array}{l}\text { Étude rétrospective } \\
\text { quantitative comparant le } \\
\text { nombre d'hospitalisations } \\
\text { pré/post services de soins } \\
\text { de soutien. }\end{array}$ & $\begin{array}{l}n=1275 \text { patients } \\
\text { en } 2011 \text { et } \\
n=1358 \text { patients } \\
\text { en 2012; inclut tous } \\
\text { les patients sous } \\
\text { chimiothérapie et } \\
\text { hospitalisés par la } \\
\text { suite en } 2011 \text { (pré } \\
\text { services de soins de } \\
\text { soutien) ou en } 2012 \\
\text { (post services de } \\
\text { soins de soutien). }\end{array}$ & $\begin{array}{l}\text { Le nombre de patients recevant } \\
\text { un traitement contre le cancer } \\
\text { a augmenté de } 6,5 \% \text {, mais les } \\
\text { hospitalisations ont diminué de } \\
3,2 \% \text { ( } p=.024) \text { avec l'ouverture } \\
\text { du centre de services de soins de } \\
\text { soutien; considéré comme une } \\
\text { mesure indirecte de l'efficacité } \\
\text { du service de soins de soutien. } \\
\text { Les visites à l'urgence ont } \\
\text { également diminué de } 5 \% \\
(p=.018) \text {. }\end{array}$ & $\begin{array}{l}\text { Année d'ouverture : } 2012 \\
\text { Personnel : cancérologues } \\
\text { médicaux } \\
\text { Horaire : Du lundi au samedi } \\
\text { le matin, mais peut se } \\
\text { poursuivre en après-midi. }\end{array}$ & $\begin{array}{l}\text { Comparaison des données d'une } \\
\text { période de } 12 \text { mois en } 2011 \text { à une } \\
\text { période de } 9 \text { mois en 2012; des } \\
\text { périodes de comparaison égales } \\
\text { peuvent avoir conduit à des } \\
\text { résultats plus significatifs. }\end{array}$ \\
\hline $\begin{array}{l}\text { Kuo et al. } \\
\text { (2017) } \\
\text { Australie }\end{array}$ & $\begin{array}{l}\text { Évaluer la prestation } \\
\text { rapide de conseils et } \\
\text { de soins aux patients } \\
\text { atteints de cancer dans } \\
\text { la Rapid Assessment } \\
\text { Clinic (RAC) versus le } \\
\text { service des urgences. }\end{array}$ & $\begin{array}{l}\text { Examen rétrospectif } \\
\text { quantitatif des dossiers } \\
\text { cliniques de septembre } 2013 \\
\text { à juin } 2014 \text { pour la RAC et le } \\
\text { service des urgences. }\end{array}$ & $\begin{array}{l}\text { La RAC a évalué } \\
n=217 \text { patients } \\
\text { sous chimiothérapie, } \\
\text { et le service des } \\
\text { urgences a évalué } \\
n=287 \text { patients } \\
\text { (152 étaient sous } \\
\text { chimiothérapie et } \\
\text { admissibles pour } \\
\text { être vus à la RAC). }\end{array}$ & $\begin{array}{l}\text { La RAC par rapport au service } \\
\text { des urgences avait des délais } \\
\text { d'examen nettement plus courts } \\
\text { (3,1 vs } 9,7 \mathrm{~h}, p<.00001) \text { et des } \\
\text { taux d'hospitalisation inférieurs } \\
(14,3 \% \text { vs } 69,1 \%, p<.00001) \\
\text { avec des séjours hospitaliers plus } \\
\text { courts }(6,5 \text { vs } 9,5 \text { jours, } p=.013) \text {. }\end{array}$ & $\begin{array}{l}\text { Année d'ouverture : } 2013 \\
\text { Personnel : Médecin- } \\
\text { stagiaire en oncologie } \\
\text { avancée et infirmière en } \\
\text { oncologie; avec infirmières } \\
\text { assurant un triage par } \\
\text { téléphone. } \\
\text { Horaire : En semaine, jours } \\
\text { non spécifiés, de } 9 \mathrm{~h} \text { à } 17 \mathrm{~h} \text {. }\end{array}$ & $\begin{array}{l}\text { Les délais d'attente pour l'examen } \\
\text { médical à la RAC avaient une } \\
\text { documentation incomplète, ce qui } \\
\text { a entraîné l'exclusion de données } \\
\text { de l'analyse, comme en témoigne } \\
\text { le large intervalle de confiance. } \\
\text { Taille réduite de l'échantillon - la } \\
\text { fiabilité des résultats bénéficierait } \\
\text { d'une période de revue } \\
\text { rétrospective plus longue. }\end{array}$ \\
\hline $\begin{array}{l}\text { Majem } \\
\text { et al. (2007) } \\
\text { Espagne }\end{array}$ & $\begin{array}{l}\text { Évaluer les procédures } \\
\text { et les résultats des } \\
\text { patients qui fréquentent } \\
\text { une unité d'oncologie } \\
\text { sur la toxicité aiguë } \\
\text { pour la prise en charge } \\
\text { de la toxicité de la } \\
\text { chimiothérapie. }\end{array}$ & $\begin{array}{l}\text { Étude rétrospective } \\
\text { quantitative de la base } \\
\text { de données de l'unité } \\
\text { d'oncologie depuis sa } \\
\text { création en février } 1999 \\
\text { jusqu'en septembre } 2001 .\end{array}$ & $\begin{array}{l}n=2007 \text { contacts } \\
\text { avec l'unité } \\
\text { d'oncologie par } \\
1126 \text { patients en } \\
\text { chimiothérapie. }\end{array}$ & $\begin{array}{l}521 \text { contacts ( } 51,4 \% \text { ont été pris } \\
\text { en charge par téléphone, mais } \\
427 \text { contacts ( } 42 \% \text { ) ont dû se } \\
\text { présenter à l'unité d'oncologie } \\
\text { pour un examen; } 59 \text { contacts } \\
\text { ont été envoyés directement } \\
\text { à l'urgence. } 209 \text { contacts } \\
\text { (49\%) ont été ultérieurement } \\
\text { hospitalisés, et tous les autres } \\
\text { sont rentrés chez eux. }\end{array}$ & $\begin{array}{l}\text { Année d'ouverture: } 1999 \\
\text { Personnel : Oncologue } \\
\text { médical et infirmière } \\
\text { spécialisée; premier contact } \\
\text { par triage téléphonique } \\
\text { avec l'infirmière. } \\
\text { Horaire : Lundi au vendredi, } \\
\text { de } 8 \text { h à } 17 \mathrm{~h} \text {. }\end{array}$ & $\begin{array}{l}\text { Analyse comparative avec les } \\
\text { admissions à l'urgence et les } \\
\text { hospitalisations avant l'OATU } \\
\text { permettrait de renforcer la } \\
\text { conception de l'étude et le niveau } \\
\text { de données probantes. }\end{array}$ \\
\hline
\end{tabular}




\begin{tabular}{|c|c|c|c|c|c|c|}
\hline $\begin{array}{l}\text { Meisenberg } \\
\text { et al. (2014) } \\
\text { États-Unis }\end{array}$ & $\begin{array}{l}\text { Mesurer la disposition } \\
\text { des patients de la } \\
\text { Supportive Care Clinic } \\
\text { (SCC) comparativement } \\
\text { au service des urgences, } \\
\text { et l'effet de la SCC sur les } \\
\text { visites à l'urgence et les } \\
\text { hospitalisations pour le } \\
\text { traitement. }\end{array}$ & $\begin{array}{l}\text { Étude rétrospective } \\
\text { quantitative des patients } \\
\text { fréquentant la SCC depuis } \\
\text { sa création en juin } 2012 \\
\text { pendant } 11 \text { mois. }\end{array}$ & $\begin{array}{l}\mathrm{n}=340 \text { visites } \\
\text { à la SCC par } \\
330 \text { patients } \\
\text { uniques souffrant } \\
\text { de symptômes liés } \\
\text { cancer. }\end{array}$ & $\begin{array}{l}\text { Les admissions pour le contrôle } \\
\text { des symptômes ont diminué de } \\
39 \text { à } 27 \text { par mois, une diminution } \\
\text { de } 31 \% \text {. Estimation de la } \\
\text { prévention des visites à l'urgence } \\
\text { de } 66 \text {. }\end{array}$ & $\begin{array}{l}\text { Année d'ouverture : } 2012 \\
\text { Personnel : clinique dirigée } \\
\text { par une infirmière autorisée } \\
\text { et intégrée dans un cabinet } \\
\text { d'oncologie. Premier contact } \\
\text { par triage téléphonique ou } \\
\text { médecin de garde après les } \\
\text { heures de bureau. } \\
\text { Horaire : Lundi au vendredi, } \\
\text { avant } 16 \mathrm{~h} 30 \text {. }\end{array}$ & $\begin{array}{l}\text { Les patients étaient parfois évalués } \\
\text { le lendemain, n'offrant ainsi pas } \\
\text { un niveau de soins similaire à celui } \\
\text { des urgences. Diminution des } \\
\text { admissions à l'urgence estimée } \\
\text { plutôt que mesurée. Une période } \\
\text { d'examen inférieure à } 12 \text { mois et } \\
\text { un échantillon de petite taille } \\
\text { réduisent la fiabilité des données. }\end{array}$ \\
\hline $\begin{array}{l}\text { Reugg } \\
\text { (2013) } \\
\text { États-Unis }\end{array}$ & $\begin{array}{l}\text { Décrire I'Immediate } \\
\text { Care Center (ICC) pour } \\
\text { les patients et comparer } \\
\text { les principales plaintes } \\
\text { prises en charge au sein } \\
\text { de l'ICC en } 2010 \text { et } 2014 .\end{array}$ & $\begin{array}{l}\text { Étude rétrospective } \\
\text { quantitative pour } \\
\text { l'assurance qualité. } \\
\text { Données examinées pour } \\
2004 \text { et } 2012 \text { afin de suivre } \\
\text { les principales raisons pour } \\
\text { lesquelles les patients se } \\
\text { sont rendus à l'ICC. }\end{array}$ & 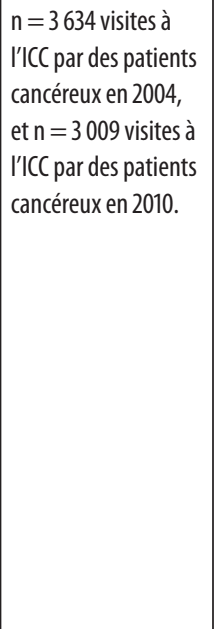 & $\begin{array}{l}\text { En 2004, les cinq principales } \\
\text { raisons de visites étaient les } \\
\text { suivantes : déshydratation } \\
\text { (35\%), douleur aiguë (17\%), } \\
\text { anémie (12\%), nausée } \\
\text { et vomissements (11\%), } \\
\text { essoufflement (10\%) et } \\
\text { fièvre (6\%). En 2010, les } \\
\text { cinq principales raisons } \\
\text { de consultations étaient: } \\
\text { déshydrations ( } 29 \%) \text {, } \\
\text { essoufflement (12\%) douleur } \\
\text { (12\%), dérangement gastro- } \\
\text { intestinal (10\%) et fièvre (5\%). } \\
\text { La satisfaction des patients fait } \\
\text { l'objet d'un suivi trimestriel } \\
\text { constamment supérieur à } 80 \% \text {. }\end{array}$ & $\begin{array}{l}\text { Année d'ouverture : } 2004 \\
\text { Personnel : Milieu sans } \\
\text { rendez-vous dirigé par } \\
\text { une infirmière autorisée; } \\
\text { l'équipe d'oncologie } \\
\text { primaire trie initialement } \\
\text { les appels des patients. } \\
\text { Horaire : En semaine, jours } \\
\text { non spécifiés, initialement } \\
\text { une unité de } 8 \text { h avec } \\
\text { une infirmière autorisée, } \\
\text { maintenant une unité de } \\
16 \text { h avec trois infirmières } \\
\text { autorisées. }\end{array}$ & $\begin{array}{l}\text { L'ICC fonctionne pour réduire } \\
\text { les admissions non planifiées } \\
\text { à l'urgence, mais l'étude n'a } \\
\text { pas évalué l'efficacité liée à ces } \\
\text { résultats. Les cinq principales } \\
\text { raisons de consultations à l'ICC } \\
\text { comptaient pour seulement } 425 \\
\text { des } 3634 \text { visites en } 2004 \text { et pour } \\
412 \text { des } 3009 \text { visites en } 2010 \text { - } \\
\text { détails vagues fournis en lien avec } \\
\text { les autres raisons de consultations. }\end{array}$ \\
\hline $\begin{array}{l}\text { Schlamb } \\
\text { (2014) } \\
\text { Canada }\end{array}$ & $\begin{array}{l}\text { Démontrer l'efficacité } \\
\text { d'un centre de } \\
\text { soins d'urgence en } \\
\text { cancérologie dans } \\
\text { les } 10 \text { premiers mois } \\
\text { d'activité. }\end{array}$ & $\begin{array}{l}\text { Ce n'est pas une étude - } \\
\text { revue rétrospective des } \\
\text { tableaux pour les nouvelles } \\
\text { activités du centre de soins } \\
\text { d'urgence en cancérologie } \\
\text { de novembre } 2013 \text { à } \\
\text { septembre } 2014 .\end{array}$ & $\begin{array}{l}\text { Nombre total de } \\
\text { patients dans } \\
\text { les cliniques et } \\
\text { volumes d'assistance } \\
\text { téléphonique dans } \\
\text { les nouveaux centres } \\
\text { de soins d'urgence } \\
\text { en cancérologie, } \\
\text { et disposition des } \\
\text { patients lors du } \\
\text { congé. }\end{array}$ & $\begin{array}{l}\text { Sur une période de } 10 \text { mois, } \\
\text { volume de patients de } 2966, \\
\text { clinique et services de soutien } \\
\text { téléphonique; } 457 \text { patients } \\
\text { (41,5\% d'augmentation) } \\
\text { transférés vers une zone de } \\
\text { traitement systémique opposé } \\
\text { à l'hospitalisation; } 110 \text { patients } \\
\text { (0,25\% de réduction) transférés } \\
\text { à l'urgence; et } 6 \text { patients } \\
\text { hospitalisés. }\end{array}$ & $\begin{array}{l}\text { Année d'ouverture : } 2013 \\
\text { Personnel : } 3 \text { infirmières } \\
\text { autorisées, rôles en } \\
\text { alternance avec triage, } \\
\text { évaluation et triage } \\
\text { téléphonique. Une } \\
\text { infirmière praticienne, un } \\
\text { omnipraticien en oncologie, } \\
\text { un oncologue médical. } \\
\text { Horaire: Lundi au vendredi, } \\
\text { de } 9 \mathrm{~h} \text { à } 17 \mathrm{~h} \text {. }\end{array}$ & $\begin{array}{l}\text { Il ne s'agit pas d'une étude, } \\
\text { mais plutôt de paramètres de } \\
\text { données recueillies présentées } \\
\text { en format PowerPoint; manque } \\
\text { d'analyse statistique et d'analyse } \\
\text { comparative avec les visites à } \\
\text { I'urgence pendant la même } \\
\text { période de collecte de données. }\end{array}$ \\
\hline $\begin{array}{l}\text { Sitzes- } \\
\text { Determann } \\
(2009) \\
\text { États-Unis }\end{array}$ & $\begin{array}{l}\text { Décrire un nouveau } \\
\text { service (centre de } \\
\text { soins d'urgence en } \\
\text { cancérologie) qui offrira } \\
\text { des soins de soutien aux } \\
\text { patients en oncologie } \\
\text { en tout temps, et } \\
\text { processus utilisé pour } \\
\text { élaborer les paramètres } \\
\text { d'évaluation, y compris } \\
\text { les fonctions du centre, } \\
\text { l'utilisation et les } \\
\text { activités. L'ouverture } \\
\text { prévue était au } \\
\text { printemps } 2009 \text {. }\end{array}$ & $\begin{array}{l}\text { Méthodologie qualitative } \\
\text { de recherche-action avec } \\
\text { méthodologie allégée } \\
\text { utilisant les principes de } \\
\text { gestion A3. Évaluation } \\
\text { de deux groupes afin de } \\
\text { répondre aux besoins des } \\
\text { parties prenantes internes } \\
\text { et externes et de planifier le } \\
\text { maintien et la croissance du } \\
\text { centre de soins d'urgence en } \\
\text { cancérologie. }\end{array}$ & $\begin{array}{l}\text { Un événement de } \\
\text { planification des } \\
\text { parties prenantes } \\
\text { a été organisé } \\
\text { pour rassembler } \\
\text { les indicateurs } \\
\text { réglementaires, de } \\
\text { conformité et de } \\
\text { qualité identifiés } \\
\text { par les employés de } \\
\text { l'hôpital ( } \mathrm{n}=27) \\
\text { pour cerner les } \\
\text { besoins des patients, } \\
\text { les opinions et les } \\
\text { idées. }\end{array}$ & $\begin{array}{l}\text { Les entretiens avec les patients } \\
\text { ont révélé deux thèmes } \\
\text { principaux définissant leurs } \\
\text { besoins: peur et anxiété ainsi } \\
\text { que fatigue - les moteurs à } \\
\text { l'origine de nombreux besoins } \\
\text { et désirs des patients. Linclusion } \\
\text { de la voix des patients aidera } \\
\text { le centre de soins d'urgence } \\
\text { en cancérologie à fournir de } \\
\text { meilleurs soins et à satisfaire les } \\
\text { patients. }\end{array}$ & $\begin{array}{l}\text { Année d'ouverture : } 2009 \\
\text { Personnel : Infirmières } \\
\text { autorisées, infirmières } \\
\text { praticiennes, techniciens en } \\
\text { soins aux patients; soutien } \\
\text { d'un médecin si nécessaire } \\
\text { (chercheur en oncologie de } \\
\text { garde ou médecin exerçant } \\
\text { en milieu hospitalier). } \\
\text { Horaire : En tout temps. }\end{array}$ & $\begin{array}{l}\text { La recherche a été menée avant } \\
\text { l'inauguration du centre, il serait } \\
\text { donc difficile de déterminer les } \\
\text { besoins non comblés des patients } \\
\text { avant l'utilisation de la clinique. }\end{array}$ \\
\hline
\end{tabular}


Ruegg, 2013; Sitzes-Determann, 2009), quatre venaient de l'Europe (Antonuzzo et al., 2017; Aprile et al., 2013; Clarke et al., 2015; Majem et al., 2007) et autant de l'Australie (Kuo et al., 2017; Livingston et al., 2011; McKenzie et al., 2011; van der Meer et al., 2016), et trois du Canada (Barbera et al., 2013; Prystajecky et al., 2012; Schlamb, 2014). Toutes les études ont été publiées entre 2007 et 2017, unr majorité $(n=11)$ ayant été réalisées au cours des cinq dernières années (Antonuzzo et al., 2017; Aprile et al., 2013; Barbera et al., 2013; Brooks et al., 2016; Clarke et al., 2015; Coyle et al., 2013; Kuo et al., 2017; Meisenberg et al., 2014; Ruegg, 2013; Schlamb, 2014; van der Meer et al., 2016).

Contrôle de la qualité. Une lacune considérable relevée dans toutes les études est le manque de collecte de données prospectives pour assurer une mesure adéquate des variables souhaitées. En fait, seules deux études ne s'appuyaient pas exclusivement sur des données rétrospectives (Brooks et al., 2016; Clarke et al., 2015). L'impossibilité d'obtenir des mesures variables spécifiques est apparue lorsqu'aucune étude n’a été trouvée pour mesurer directement les effets de la mise en place d'un centre de soins d'urgence en cancérologie sur les taux de visites à l'urgence et les hospitalisations, ni les économies directes associées à ce changement dans la prestation des soins.

Comparer le traitement reçu par les patients externes présentant des symptômes aigus du cancer dans les services des urgences comparativement aux centres de soins d'urgence en cancérologie

La première grande question de recherche de l'étude était : Quel est l'état de la science concernant les centres de soins d'urgence en cancérologie, et quelles sont les différences si on les compare aux services d'urgences en matière de traitement prodigué aux patients externes présentant des symptômes aigus du cancer?

Les résultats présentés aux tableaux 4 et 5 ont permis de recenser les domaines de recherche dominants en matière de traitement des patients externes présentant des symptômes aigus du cancer dans les services d'urgence et les centres de soins d'urgence en cancérologie et de relever les lacunes dans les recherches et la littérature scientifique actuelles. L'analyse et synthèse des données résultant d'une comparaison du traitement du patient externe présentant des symptômes aigus du cancer dans deux contextes de soins différents a fait ressortir trois grands thèmes : le rapport coût-efficacité, l'amélioration de la sécurité, et les soins centrés sur le patient. La prochaine section du document présentera de manière narrative les résultats relatifs à la question centrale initiale, en identifiant les thèmes et sous-thèmes de la littérature examinée.

\section{Rapport coût-efficacité des soins en cancérologie.}

Centres de soins d'urgence en cancérologie. L'introduction des centres de soins d'urgence en cancérologie s'est avérée rentable compte tenu de la diminution conséquente des visites non prévues à l'urgence et des hospitalisations. Une étude américaine a estimé à 66 le nombre de visites à l'urgence évitées (Meisenberg et al., 2014); quatre autres études supposaient que l'introduction des centres de soins d'urgence en cancérologie avait entraîné une diminution des visites à l'urgence (Aprile et al., 2013; Kuo et al., 2017; Majem et al., 2007; Ruegg, 2013). Au Canada, un rapport en ligne a indiqué que le centre de soins d'urgence en cancérologie de Winnipeg a évalué 3000 patients sur une période de dix mois, ce qui a vraisemblablement détourné ces patients des urgences engorgées (Schlamb, 2014). L'une des principales lacunes de ce rapport canadien et des cinq autres études citées ci-dessus est le manque de données comparatives sur les services des urgences pour déterminer si ces centres de soins d'urgence en cancérologie réussissaient effectivement à réduire directement les visites à l'urgence pour les patients présentant des symptômes aigus du cancer. En Italie toutefois, des chercheurs ont établi une corrélation directe et une réduction cliniquement significative de $5 \%$ des visites à l'urgence au cours des douze premiers mois de fonctionnement du centre de soins d'urgence en cancérologie (Antonuzzo et al., 2017).

La diminution du nombre d'hospitalisations était très variable, allant d'une diminution de 3,2\% (Antonuzzo et al., 2017) à 54,8\% (Kuo et al., 2017) avec des diminutions associées du nombre de jours d'hospitalisation de $15,1 \%$ et de $42,9 \%$, respectivement. Coyle et collègues (2013) ont commenté les différences entre les taux de visites aux centres de soins d'urgence en cancérologie et ceux aux urgences - 34 \% versus $83 \%$ respectivement, mais la signification clinique n’a pas été précisée. Dernier élément d'intérêt, Majem et collègues (2007) ont remarqué que $49 \%$ des patients des centres de soins d'urgence en cancérologie avaient été hospitalisés, ce qui indique que bien que les visites à l'urgence puissent avoir être évitées, l'hospitalisation a été inévitable pour certains patients en fonction des symptômes qu'ils présentaient.

Meilleure utilisation des services d'urgence. L'analyse des scores de l'échelle de triage et de gravité (ÉTG) permet d'identifier les symptômes aigus du cancer qui sont mieux pris en charge dans un cadre de soins externes, notamment les scores de l'ÉTG (voir tableau 1) qui sont de trois ou plus (délai de prise en charge thérapeutique de 30 minutes ou plus). Dans la littérature, les catégories de l'ÉTG où sont fréquemment classés les patients cancéreux qui consultent à l'urgence étaient les niveaux 3 et 4 . Au Canada, ça représente de 57,0-66,3\% des patients cancéreux qui consultent à l'urgence (Barbera et al., 2013; Prystajecky et al., 2012), et de 75,5-82,8 \% en Australie (Livingston et al., 2011; van der Meer et al., 2016). Le service des urgences devrait être réservé si possible aux patients en danger de mort ou présentant des symptômes nécessitant des soins médicaux très urgents, afin de limiter l'engorgement dû à la présentation de symptômes ne justifiant pas le recours aux urgences. Les études canadiennes ont également montré une forte proportion de résultats à l'ÉTG de niveau $2(28,4-41,0 \%$ des patients atteints de cancer se rendant aux urgences) (Barbera et al., 2013; Prystajecky et al., 2012). Les scores du niveau 1 et 2 selon l'ÉTG visent des délais d'intervention inférieurs à 15 minutes et constituent une utilisation appropriée des services des urgences.

Optimiser le rôle des prestataires de soins en oncologie. Brooks et collègues (2016) ont observé les effets de l'intégration d'un oncologue d'urgence pendant en soirée. Bien que créative, cette approche n'a pas été jugée rentable ni efficace pour réduire les taux d'hospitalisation (Brooks et al., 2016). Subjectivement, selon les oncologues participants, $27 \%$ des visites à l'urgence pourraient être prises en charge en milieu de soins externes sans prise en charge par le service d'urgence, et $19 \%$ des hospitalisations pourraient être évitées (Brooks 
et al., 2016). Par ailleurs, les centres de soins d'urgence en cancérologie qui ont des infirmières praticiennes en oncologie favorisent un bon rendement économique en réduisant le coût des consultations, le nombre de consultations avec les oncologues et les interruptions de protocoles de chimiothérapie (Meisenberg et al., 2014; Ruegg, 2013).

Amélioration de la sécurité des patients atteints de cancer. Expertise ciblée en oncologie. La majorité des patients qui reçoivent un diagnostic de cancer seront confrontés à des parcours de soins fragmentés en raison des soins souvent dispensés par plusieurs établissements et services dans un centre de cancérologie. Cette complexité peut avoir un effet négatif sur la sécurité et la qualité des soins (Brooks et al., 2016). L'introduction des centres de soins d'urgence en cancérologie réduit cette fragmentation des soins et en favorise la continuité. Elle se caractérise en outre par une différence de temps significative pour l'initiation des antibiotiques chez les patients atteints de fièvre neutropénique. Au centre de soins d'urgences de l'Ohio, le délai d'administration des antibiotiques n'est que de quelques minutes par rapport à une moyenne de 1,7 heure aux urgences (Ruegg, 2013). De même, le temps total passé dans un centre de soins d'urgence en cancérologie était inférieur de $48 \%$ au temps passé dans un service des urgences, selon Coyle et collègues (2013). Cette différence significative équivaut à un temps total moyen consacré à l'examen des patients de 3,1 heures dans un centre de soins d'urgence en cancérologie contre 9,7 heures dans un service des urgences (Kuo et al., 2017). Brooks et collègues (2016) ont également indiqué qu'une intervention précoce de l'oncologue après l'apparition des symptômes du cancer permettait d'améliorer la coordination des soins pour les patients, un avantage supplémentaire des centres de soins d'urgence en cancérologie. La diminution des taux d'hospitalisation des centres de soins d'urgence en cancérologie (34\%) par rapport à l'urgence $(83 \%)$ a également été associée à une diminution du risque de mortalité et à une amélioration de la qualité de vie (Coyle et al., 2013). Enfin, les centres de soins urgents en cancérologie ont été associés à des durées d'hospitalisation plus courtes pour les patients cancéreux par rapport à l'urgence (Antonuzzo et al., 2017; Kuo et al., 2017; Meisenberg et al., 2014).

Perception des patients à l'égard des services des urgences. Une autre interrogation relative à la sécurité était la réticence des patients à consulter à l'urgence. McKenzie et collègues (2011) ont mentionné plusieurs raisons pour cette résistance possible : ne pas vouloir déranger le personnel très occupé, la possible difficulté d'accès, les longues périodes d'attente une fois arrivés à l'urgence, l'optimisme que les symptômes disparaîtront d'eux-mêmes. Quelques études ont montré que les patients nieront leurs symptômes pour éviter une hospitalisation possible en raison de mauvaises expériences passées ou pour éviter de se retrouver dans un service d'urgence (Clarke et al., 2015; Sitzes-Determann, 2009). Comme décrit dans la littérature, les patients sentent que le personnel des urgences s'appuie largement sur eux pour fournir de l'information associée à leur diagnostic et à leur traitement (Sitzes-Determann, 2009). Les auteurs expliquent cette dépendance du personnel des services des urgences aux explications des patients par le manque d'accessibilité aux dossiers médicaux inter-établissements, ce qui conduit à une diminution de la sécurité perçue par les patients accédant à l'urgence pour le traitement des symptômes aigus du cancer (Sitzes-Determann, 2009).

\section{Soins de grande qualité centrés sur le patient.}

Comprendre les symptômes du cancer. Pour prodiguer des soins de grande qualité, il faut répondre à la fois aux besoins connus et potentiels des patients. Une étude canadienne de Barbera et collègues (2013) a démontré l'efficacité de la prévision des visites à l'urgence par le suivi des scores des symptômes signalés par les patients à l'aide de l'Échelle d'évaluation des symptômes d'Edmonton utilisé lors des visites de routine en oncologie. Plusieurs symptômes évalués (ex. douleur, malaise, nausée, somnolence, essoufflement) ont été associés à des visites à l'urgence dans la semaine suivant l'évaluation des symptômes (Barbera et al., 2013). Les résultats ont également démontré que ces « signes généraux étaient associés à une probabilité encore plus élevée de recours à l'urgence » (p. 427), indiquant une mauvaise utilisation des ressources en milieu de soins externes (Barbera et al., 2013).

Services élargis de lutte contre le cancer. Dans le monde entier, les centres de soins externes en cancérologie sont généralement ouverts du lundi au vendredi, de jour. Trois études qui rapportaient le moment de la journée où les patients atteints de cancer avaient le plus souvent eu recours aux services d'urgence sont venues confirmer que plus de la moitié des visites ont lieu en semaine, pendant la journée (Barbera et al., 2013; Livingston et al., 2011; Mayer et al., 2011). Selon Brooks et collègues (2016), les services de cancérologie externes devraient élargir leurs heures d'ouverture plus longues, par exemple en fin d'après-midi ou en début de soirée. Quatre des sept centres de soins d'urgence en cancérologie examinés offraient un horaire prolongé en soirée (Aprile et al., 2013; Majem et al., 2007; Ruegg, 2013) tandis que deux centres des États-Unis se targuent d'avoir des centres de soins d'urgence en cancérologie ouverts en tout temps (Coyle et al., 2013). Des sept centres de soins d'urgence en cancérologie, seulement un n'offrait pas systématiquement des rendez-vous le jour même (Meisenberg et al., 2014). Un établissement de soins externes peut également avoir une plus grande capacité à offrir des procédures spécialisées le jour même, telles que des thoracentèses thérapeutiques ou des paracentèses (Brooks et al., 2016). Au centre des soins d'urgence en cancérologie en Ohio, les infirmières praticiennes accomplissent ces procédures et renvoient les patients chez eux quelques heures plus tard (Ruegg, 2013). En Espagne, des médecins sont également présents dans les centres de soins d'urgence en cancérologie pour accomplir ces tâches (Majem et al., 2007).

\section{Activités actuelles des centres de soins d'urgence en cancérologie}

La deuxième question de recherche abordée dans cette revue intégrative était : Où sont les centres de soins d'urgences en cancérologie dans le monde, et que sait-on de leurs activités cliniques et modèles de dotation?

Dans la documentation comprise dans cette revue, neuf centres de soins d'urgence en cancérologie ont été décrits en détail. Ces neuf centres sont actuellement en opération dans différentes parties du monde, notamment en Espagne 
(Majem et al., 2007) au Canada (Schlamb, 2014), aux ÉtatsUnis (Coyle et al., 2013; Meisenberg et al., 2014; Ruegg, 2013; Sitzes-Determann, 2009), en Italie (Antonuzzo et al., 2017; Aprile et al., 2013) et en Australie (Kuo et al., 2017). Les neuf centres de soins d'urgence en cancérologie sont décrits au tableau 5. On y constate que le nom des centres, les activités et le personnel varient beaucoup. Historiquement, le premier centre à ouvrir ses portes en 1999 a été une unité d'oncologie sur la toxicité aiguë, en Espagne (Majem et al., 2007). Les centres les plus récents ont été ouverts en 2013, avec le Rapid Assessment Clinic en Australie (Kuo et al., 2017), et lune clinique de soins urgents en cancérologie à Winnipeg, au Canada. Six centres de soins d'urgence en cancérologie sont dotés d'oncologues médicaux (Antonuzzo et al., 2017; Aprile et al., 2013; Coyle et al., 2013; Kuo et al., 2017; Majem et al., 2007; Schlamb, 2014); les trois autres emploient des infirmières praticiennes (Meisenberg et al., 2014; Ruegg, 2013; Sitzes-Determann, 2009). La plupart des centres font également appel à une infirmière autorisée. Les heures d'ouverture de tous les centres de soins d'urgence en cancérologie étaient variables et allaient des heures du matin seulement (Antonuzzo et al., 2017) aux heures régulières de travail de jour (Kuo et al., 2017; Majem et al., 2007; Meisenberg et al., 2014; Schlamb, 2014), certains étant ouverts pendant 12 heures (Aprile et al., 2013), 16 heures (Ruegg, 2013), ou 24 heures (Coyle et al., 2013; Sitzes-Determann, 2009). La plupart des centres de soins d'urgence en cancérologie sont ouverts en semaine, mais un centre est ouvert le samedi (Antonuzzo et al., 2017) et deux autres fonctionnent en tout temps (Coyle et al., 2013; Sitzes-Determann, 2009). Enfin, cinq centres de soins d'urgence en cancérologie (Kuo et al., 2017; Majem et al., 2007; Meisenberg et al., 2014; Ruegg, 2013; Schlamb, 2014) ont mentionné l'utilisation du triage par téléphone comme méthode de contact initial chez les patients potentiels avant la visite. Le triage téléphonique permet d'éviter qu'un patient présentant des symptômes ne nécessitant pas une vist=ite au centre se présente et permet une prise en charge thérapeutique à domicile à l'aide du soutien téléphonique, ou une recommandation d'aller directement à l'urgence (Kuo et al., 2017; Meisenberg et al., 2014).

\section{DISCUSSION}

Lobjectif de cette revue intégrative de la littérature était de répondre à deux grandes questions de recherche : 1) Quel est l'état de la science concernant les centres de soins d'urgence en cancérologie, et quelles sont les différences si on les compare aux services d'urgences en matière de traitement prodigué aux patients externes présentant des symptômes aigus du cancer? 2) Où sont les centres de soins d'urgences en cancérologie dans le monde, et que sait-on de leurs activités cliniques et modèles de dotation? Trois grands thèmes sont ressortis des données en réponse à la première grande question de recherche : le rapport coût-efficacité, l'amélioration de la sécurité et les soins centrés sur le patient. La deuxième question a permis de déterminer où les centres de soins d'urgence en cancérologie opèrent actuellement dans le monde et les modèles de dotation en personnel utilisés par ces centres.
Un bon rapport coût-efficacité a été atteint après l'introduction des centres de soins d'urgence en cancérologie en réduisant les visites à l'urgence, les hospitalisations, et, le cas échéant, la durée du séjour (Antonuzzo et al., 2017; Aprile et al., 2013; Coyle et al., 2013; Kuo et al., 2017; Majem et al., 2007; Meisenberg et al., 2014; Ruegg, 2013; Schlamb, 2014). La prestation dans un milieu de soins ambulatoires a également permis de réduire les coûts globaux liés au traitement. De même, les patients ont connu moins d'interruptions de traitement du cancer ou de visites chez l'oncologue liées au traitement des symptômes aigus du cancer dans les centres de soins d'urgence en cancérologie, ce qui entraîne une réduction indirecte des dépenses liées au cancer (Meisenberg et al. 2014; Ruegg, 2013). Plus précisément, les oncologues travaillant dans un centre de soins d'urgence en cancérologie ont été mieux à même de combler les lacunes entre les besoins immédiats des patients et le traitement du cancer en cours en raison de leur familiarité implicite avec les processus des centres de soins d'urgence en cancérologie. Enfin, les économies peuvent être encore accrues en faisant appel à des infirmières praticiennes spécialisées dans les centres de soins d'urgence en cancérologie, par opposition aux oncologues médicaux, sans compromettre la sécurité des patients ou la qualité des soins (Meisenberg et al., 2014; Ruegg, 2013).

Les centres de soins d'urgence en cancérologie ont également amélioré la sécurité des patients atteints de cancer en apportant un contrepoint aux expériences et perceptions des patients liées à l'utilisation des services des urgences. Comme les centres de soins d'urgence en cancérologie se trouvent dans des centres de soins externes en cancérologie, l'accès aux dossiers médicaux des patients est immédiat et le volume de patients accueilli par ces centres étant plus faible qu'à l'urgence, la rapidité et la coordination des soins aux patients atteints de cancer sont meilleures (Brooks et al., 2016; Coyle et al., 2013; Kuo et al., 2017; Ruegg, 2013). Bien que ce point n'ait pas fait l'objet d'une étude directe, les centres de soins d'urgence en cancérologie contribuent probablement à annuler certaines des perceptions défavorables des patients associées à l'urgence, telles que la forte dépendance du personnel à l'égard des patients concernant leur diagnostic et leur plan de traitement du cancer, ou le déni des symptômes pour éviter de longs délais d'attente à l'urgence ou d'incommoder le personnel (Clarke et al., 2015; McKenzie et al., 2011; SitzesDetermann, 2009). Les obstacles étant moins nombreux, les patients peuvent rechercher plus rapidement une intervention auprès des centres de soins d'urgence en cancérologie, ce qui contribue également à des soins plus sûrs.

Le dernier thème qui est ressorti est l'idée que les centres de soins d'urgence en cancérologie fournissent des soins de meilleure qualité, centrés sur le patient. Les centres de soins d'urgence en cancérologie offrent des rendez-vous le jour même, sans rendez-vous, ce qui ressemble aux visites à l'urgence, mais avec les avantages supplémentaires d'accéder à des spécialistes en oncologie qui connaissent le traitement de tous les types de cancers ainsi que la visite clinique s'effectue dans un lieu familier. En outre, certains centres de soins d'urgence en cancérologie ont la capacité supplémentaire 
d'effectuer des interventions spécialisées en soins externes (c'est-à-dire des thoracentèses ou des paracentèses), ce qui permet de répondre et de soulager rapidement les besoins aigus, et parfois palliatifs, des patients (Brooks et al., 2016; Majem et al., 2007; Ruegg, 2013). En fin de compte, la capacité des centres de soins d'urgence en cancérologie à offrir des soins de soutien en temps utile et à réagir aux signes généraux plus rapidement, contrairement à l'urgence, améliorera la qualité de vie globale des patients et des familles vivant avec le cancer (Barbera et al., 2013).

Bien que les services des urgences aient su fournir un service crucial aux patients présentant des symptômes aigus de cancer, l'utilisation continue de ce modèle de soins pour les patients en oncologie reste coûteuse, comporte des risques de santé, et demeure une approche de soins peu centrée sur le patient. Sur tous les continents, l'efficacité et les dépenses de santé sont une priorité absolue, tant pour les prestataires que pour les patients. L'émergence de ces centres répondrait à ces deux priorités. Cependant, la faible quantité de littérature disponible pour soutenir l'adoption de ce nouveau modèle de soins est problématique. Bien que le premier centre documenté ait ouvert en 1999, les résultats cliniques n'ont pas été signalés dans la littérature avant 2007 (Majem et al., 2007). Au cours de la dernière décennie, seulement huit études axées sur les centres de soins d'urgence en cancérologie ont pu être recensées (Antonuzzo et al., 2017; Aprile et al., 2013; Coyle et al., 2013; Kuo et al., 2017; Meisenberg et al., 2014; Ruegg, 2013; Schlamb, 2014; SitzesDetermann, 2009). Selon ces études, les centres de soins d'urgence en cancérologie offrent une nouvelle alternative pour le traitement des patients externes présentant des symptômes avec, selon les premières données recueillies, une amélioration des soins aux patients par rapport à l'urgence.

\section{Limites}

Plusieurs limites méthodologiques ont influencé les résultats de cette revue intégrative. Seule la première auteure, étudiante en sciences infirmières, a passé en revue toute la littérature recensée, ce qui augmente le risque de partialité. De même, la combinaison et la complexité inhérentes à l'intégration de plusieurs méthodologies peuvent réduire la rigueur et augmenter l'inexactitude ou la partialité des résultats (Whittemore et Knafl, 2005). Il est également reconnu par Whittemore et Knafl (2005) que les méthodes d'analyse, de synthèse et de formulation de conclusions restent peu élaborées pour les revues intégratives. Toutefois, les résultats présentés constituent une introduction à un sujet original et d'actualité, à savoir les soins de soutien dans les centres de soins d'urgence en cancérologie pour les patients externes présentant des symptômes aigus du cancer.

\section{Implications pour la pratique infirmière et la recherche future}

Cette revue intégrative a des implications sur la pratique infirmière. Améliorer l'expérience du patient en ce qui concerne les symptômes du cancer devrait commencer lors des visites de routine dans les centres de cancérologie, par des évaluations appropriées et détaillées de même qu'une prise en charge précoce des symptômes non soulagés (ex. douleur, détresse respiratoire, nausée ou vomissement) fréquemment traités dans les services des urgences (Barbera et al., 2013; Mayer et al., 2011; van der Meer et al., 2016). Certains symptômes spécifiques tels que la douleur, la nausée ou l'essoufflement, ainsi que divers signes généraux liés au bien-être, à la fatigue et à l'appétit sont prévisibles et associés aux visites ultérieures dans les services des urgences (Barbera et al., 2013). Par conséquent, les symptômes spécifiques susmentionnés devraient être ciblés dans les milieux de soins externes par l'essai actif de stratégies de traitement prophylactique et préventif (Livingston et al., 2011), alors que les dignes généraux peuvent nécessiter des interventions sur le plan psychosocial ou alimentaire, ainsi que des discussions sur les objectifs des soins pour aider à éviter d'éventuelles visites à l'urgence. La possibilité de prévoir et de prévenir ces événements indésirables courants liés au cancer était une stratégie recommandée par certains chercheurs (Aprile et al., 2013; Barbera et al., 2013). L'importance de renforcer l'éducation des patients et des soignants par rapport à l'autogestion des symptômes a également été soulignée par van der Meer et collègues (2016). D’une manière générale, les patients et les prestataires de soins « ont besoin d'information précise, cohérente et sans ambiguïté sur ce qu'il faut faire en cas de malaise ou d'état fébrile après la chimiothérapie » afin d'empêcher qu'il y ait des délais s'ils ont besoin d'aide pour traiter leurs symptômes (Clarke et al., 2015; McKenzie et al., 2011).

Le soutien téléphonique est une autre intervention relevée en tant que première mesure importante pour prévenir d'éventuelles visites à l'urgence pour les patients cancéreux (Majem et al., 2007). Les infirmières spécialisées en oncologie formées pour filtrer et trier correctement les appels téléphoniques contribuent à garantir que les patients reçoivent les soins appropriés au meilleur endroit possible (soins auto-administrés à domicile, en personne chez un médecin de famille, à l'urgence ou dans un centre de soins d'urgence en cancérologie). En retour, cela optimiserait les ressources des soins de santé (Meisenberg et al., 2014). Comme l'a fait remarquer Ruegg (2013), les infirmières et les « infirmières praticiennes spécialisées sont spécifiquement équipées pour la prise en charge des problèmes liés au cancer avant qu'ils ne deviennent si graves qu'ils nécessitent une hospitalisation » (p. E55). Tirer parti des forces des infirmières spécialisées en oncologie pour le traitement des symptômes aigus du cancer bénéficiera astucieusement à tous les acteurs concernés, mais surtout aux patients et à leurs soignants. En ce qui concerne les implications futures pour la recherche, l'accent mis sur les études qualitatives et les études quantitatives prospectives renforcerait les preuves existantes trouvées dans la littérature. Des efforts de recherche consacrés à l'évaluation de l'efficacité et de la rentabilité des centres de soins d'urgence en cancérologie sont nécessaires pour entraîner d'éventuels changements de pratique. De plus, la recherche qualitative sur l'expérience des prestataires de soins de santé en oncologie (c'est-à-dire les infirmières autorisées, les infirmières praticiennes et les médecins) qui travaillent dans les centres de soins d'urgence en cancérologie et les administrent, ou l'expérience des patients atteints de cancer qui sont familiers avec les deux modèles de soins, demeurent absents de la littérature. Davantage de recherche est également nécessaire pour mieux 
comprendre les processus des centres de soins d'urgence en cancérologie, tout en examinant les obstacles réels et possibles au fonctionnement de ces centres. Enfin, même si les centres de soins d'urgence en cancérologie existent au Canada, il n'existe actuellement aucune publication canadienne évaluée par des pairs concernant ce nouveau modèle. Poursuivre la recherche sur les centres de soins d'urgence en cancérologie, au Canada comme à l'étranger, est essentiel pour assurer une transition réussie du modèle actuel des services d'urgence pour les patients en cancérologie vers le modèle fort prometteur de traitement des symptômes aigus du cancer du patient externe dans les centres de soins d'urgence en cancérologie.

\section{CONCLUSION}

Tout au long de cette revue intégrative, trois grands thèmes se sont dégagés de la littérature en réponse à la première question centrale. En outre, la deuxième question de recherche a permis d'identifier les centres de soins d'urgence en cancérologie dans le monde entier et de consigner leurs activités cliniques et leurs modèles de dotation en personnel. Les thèmes dégagés indiquent que, par rapport à l'urgence, les centres favoriseraient des soins aux patients en cancérologie rentables, plus sûrs, de meilleure qualité et centrés sur le patient. Les connaissances scientifiques sur les centres de soins d'urgence en cancérologie

\section{RÉFÉRENCES}

Antonuzzo, A., Vasile, E., Sbrana, A., Lucchesi, M., Galli, L., Brunetti, I. M., Musettini, G., Farnesi, A., Biasco, E., Virgili, N., Falcone, A., \& Ricci, S. (2017). Impact of a supportive care service for cancer outpatients: Management and reduction of hospitalizations. Preliminary results of an integrated model of care. Supportive Care in Cancer, 25(1), 209-212. https://doi.org/10.1007/s00520-016-3403-z

Aprile, G., Pisa, F. E., Follador, A., Foltran, L., De Pauli, F., Mazzer, M., Lutrino, S., Sacco, C. S., Mansutti, M., \& Fasola, G. (2013). Unplanned presentations of cancer outpatients: A retrospective cohort study. Supportive Care in Cancer, 21(2), 397-404. https://doi. org/10.1007/s00520-012-1524-6

Barbera, L., Atzema, C., Sutradhar, R., Seow, H., Howell, D., Husain, A., Sussman, J., Earle, C., Liu, Y., \& Dudgeon, D. (2013). Do patient-reported symptoms predict emergency department visits in cancer patients? A population-based analysis. Annals of Emergency Medicine, 61(4), 427-437. https://doi.org/10.1016/j. annemergmed.2012.10.010

Brooks, G. A., Chen, E. J., Murakami, M. A., Giannakis, M., Baugh, C. W., \& Schrag, D. (2016). An ED pilot intervention to facilitate outpatient acute care for cancer patients. American Journal of Emergency Medicine, 34(10), 1934-938. https://doi.org/10.1016/j. ajem.2016.06.076

Canadian Cancer Statistics Advisory Committee. (2019). Canadian cancer statistics 2019. Canadian Cancer Society. https://www.cancer. ca/ /media/cancer.ca/CW/cancer\%20information/cancer\%20 101/Canadian\%20cancer\%20statistics/Canadian-Cancer-Statistics2019-EN.pdf?la=en

Clarke, R. T., Bird, S., Kakuchi, I., Littlewood, T. J., \& van Hamel Parsons, V. (2015). The signs, symptoms and help-seeking experiences of neutropenic sepsis patients before they reach hospital: A qualitative study. Supportive Care in Cancer, 23(9), 26872694. https://doi.org/10.1007/s00520-015-2631-y augmentent, mais davantage de recherche est nécessaire pour arriver à répondre aux besoins du patient de manière appropriée. Jusqu'à maintenant, il n'y a pas d'étude qualitative axée sur l'expérience des prestataires de soins oncologiques travaillant dans ces centres. Leur expérience, en plus d'une description précise des processus de fonctionnement dans les centres de soins d'urgence en cancérologie, devrait primer lors des futurs efforts de recherche. La demande d'une meilleure compréhension globale des activités et des expériences des centres de soins d'urgence en cancérologie peut contribuer à l'adoption universelle du traitement des patients externes présentant des symptômes aigus du cancer. Réduire autant que possible les consultations à l'urgence pour des symptômes aigus, mais non urgents du cancer aura du bon et contribuera à ce que tous les patients profitent même système de soins de santé et reçoivent « des soins continus de qualité, au bon endroit, au bon moment et sous la supervision des bons spécialistes » (Young et al., 2016, p. 301).

\section{REMERCIEMENTS}

Cette étude a été rendue possible en partie grâce à la bourse d'études pour le personnel en oncologie Marlene Mysak 2020 de l'Alberta Care Foundation, à la bourse Denise Melanson 2019, ainsi qu'à la bourse d'études annuelle 2019 de l'Alberta Registered Nurses Educational Trust (ARNET).

Colligan, E. M., Ewald, E., Keating, N. L., Parashuram, S., Spafford, M., Ruiz, S., \& Moiduddin, A. (2017). Two innovative cancer care programs have potential to reduce utilization and spending. Medical Care, 55(10), 873-878. https://doi.org/10.1097/ MLR.0000000000000795

Cooksley, T., \& Rice, T. (2017). Emergency oncology: Development, current position and future direction in the USA and UK. Supportive Care in Cancer, 25(1), 3-7. https://doi.org/10.1007/ s00520-016-3470-1

Coyle, Y. M., Miller, A. M., \& Paulson, R. S. (2013). Model for the costefficient delivery of continuous quality cancer care: A hospital and private-practice collaboration. Baylor University Medical Center Proceedings, 26(2), 9599. https://www.ncbi.nlm.nih.gov/ pubmed/23543960

Hassett, M. J., O’Malley, A. J., Pakes, J. R., Newhouse, J. P., \& Earle, C. C. (2006). Frequency and cost of chemotherapy-related serious adverse effects in a population sample of women with breast cancer. Journal of the National Cancer Institute, 98(16), 1108-1117. https://doi.org/10.1093/jnci/djj305

Kuo, J. C., De Silva, M., Diwakarla, C., \& Yip, D. (2017). A Rapid Access Clinic to improve delivery of ambulatory care to cancer patients. Asia-Pacific Journal of Clinical Oncology, 13(3), 179-184. https://doi. org/10.1111/ajco.12641

Livingston, P. M., Craike, M., \& Considine, J. (2011). Unplanned presentations to emergency departments due to chemotherapy induced complications: Opportunities for improving service delivery. Australasian Emergency Nursing Journal, 14(2), 62-68.

Majem, M., Galán, M., Pérez, F. J., Muñoz, M., Chicote, S., Soler, G., Navarro, M., Martínez-Villacampa, M., García del Muro, X., Dotor, E., Laquente, B., \& Germà, J. R. (2007). The Oncology Acute Toxicity Unit (OATU): An outpatient facility for improving the management of chemotherapy toxicity. Clinical and Translational Oncology, 9(12), 784-788. 
Mason, H., DeRubeis, M. B., Foster, J. C., Taylor, J. M., \& Worden, F. P. (2013). Outcomes evaluation of a weekly nurse practitionermanaged symptom management clinic for patients with head and neck cancer treated with chemoradiotherapy. Oncology Nursing Forum, 40(6), 581-586. https://doi.org/10.1188/13.ONF.40-06AP

Mayer, D. K., Travers, D., Wyss, A., Leak, A., \& Waller, A. (2011). Why do patients with cancer visit emergency departments? Results of a 2008 population study in North Carolina. Journal of Clinical Oncology, 29(19), 2683-2688. https://doi.org/10.1200/ jco.2010.34.2816

McKenzie, H., Hayes, L., White, K., Cox, K., Fethney, J., Boughton, M., \& Dunn, J. (2011). Chemotherapy outpatients' unplanned presentations to hospital: A retrospective study. Supportive Care in Cancer, 19(7), 963-969. https://doi.org/10.1007/s00520-010-0913-y

Meisenberg, B. R., Graze, L., \& Brady-Copertino, C. J. (2014). A supportive care clinic for cancer patients embedded within an oncology practice. The Journal of Community and Supportive Oncology, 12(6), 205-208.

Prystajecky, M., Lang, E., Wang, D., Simon, J., Sinnarajah, A., Martin, T. L. W., Murray, A., Hagen, N., Waller, A., Bultz, B., Carlson, L., Groff, S., McRae, A., Thomas, B. (2012). Emergency department utilization by adult cancer patients: A prospective cohort study. Academic Emergency Medicine, 19, S225-S226.

Ruegg, T. A. (2013). A nurse practitioner-led urgent care center: Meeting the needs of the patient with cancer. Clinical Journal of Oncology Nursing, 17(4), E52-E57. https://doi.org/10.1188/13.CJON. E52-E57

Schlamb, T. (2014). Urgent cancer care and the cancer helpline. http:// www.cancercare.mb.ca/resource/File/MBWCN/presenations/ Urgent_Care_Presentation-Tracy_Schlamb.pdf

Sitzes-Determann, A. L. (2009). Planning for the sustainment of a 24/7 oncology clinic: An action and evaluation research project. (Doctoral dissertation). http://ezproxy.lib.ucalgary.ca/login?url=http:// search.ebscohost.com/login.aspx?direct=true \&db=rzh\&AN=10985 $3890 \&$ site $=$ ehost-live
Specialist Unit for Review of Evidence (SURE). (2018a). Questions to assist with the critical appraisal of cohort studies. https://www.cardiff. ac.uk/__data/assets/pdf_file/0006/1142997/SURE-CA-form-forCohort_2018.pdf

Specialist Unit for Review of Evidence (SURE). (2018b). Questions to assist with the critical appraisal of qualitative studies. https://www. cardiff.ac.uk/_data/assets/pdf_file/0007/1142971/SURE-CA-formfor-Qualitative_2018.pdf

Trip, K., Boloorchi, A., \& Berman, H. (2011). Utilizing the Canadian emergency department Triage and Acuity Scale (CTAS) in an oncology urgent care clinic: The university health network experience. Canadian Oncology Nursing Journal, 21(1), 52-57. https://www.ncbi.nlm.nih.gov/pubmed/21462878

van der Meer, D. M., Weiland, T. J., Philip, J., Jelinek, G. A., Boughey, M., Knott, J., Marck, C. H., Weil, J. L., Lane, H. P., Dowling, A. J., \& Kelly, A. M. (2016). Presentation patterns and outcomes of patients with cancer accessing care in emergency departments in Victoria, Australia. Supportive Care in Cancer, 24(3), 1251-1260. https://doi. org/10.1007/s00520-015-2921-4

Vandyk, A. D., Harrison, M. B., Macartney, G., Ross-White, A., \& Stacey, D. (2012). Emergency department visits for symptoms experienced by oncology patients: A systematic review. Supportive Care in Cancer, 20(8), 1589-1599. https://doi.org/10.1007/ s00520-012-1459-y

Whittemore, R., \& Knafl, K. (2005). The integrative review: updated methodology. Journal of Advanced Nursing, 52(5), 546-553. https:// doi.org/10.1111/j.1365-2648.2005.03621.x

Young, A., Marshall, E., Krzyzanowska, M., Robinson, B., Brown, S., Collinson, F., Seligmann, J., Abbas, A., Rees, A., Swinson, D., Neville-Webbe, H., \& Selby, P. (2016). Responding to acute care needs of patients with cancer: Recent trends across continents. The Oncologist, 21(3), 301-307. https://doi.org/10.1634/ theoncologist.2014-0341 